\title{
On the Generation of Hydrodynamic Shocks by Mixed Beams and Occurrence of Sunquakes in Flares
}

\author{
Valentina Zharkova ${ }^{1} \cdot$ Sergei Zharkov ${ }^{2}$
}

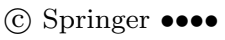

\begin{abstract}
Observations of solar flares with sunquakes by the space and groundbased instruments reveal essentially different dynamics of seismic events in different flares. Some sunquakes are found to be closely associated with the locations of hard X-ray (HXR) and white-light (WL) emission while others are located outside either of them. In this article we investigate possible sources causing a seismic response in a form of hydrodynamic shocks produced by the injection of mixed (electron plus proton) beams, discuss the velocities of these shocks, and the depths where they deposit the bulk of their energy and momentum. The simulation of hydrodynamic shocks in flaring atmospheres induced by electronrich and proton-rich beams reveals that the linear depth of the shock termination is shifted beneath the level of the quiet solar photosphere on a distance from 200 to $5000 \mathrm{~km}$. The parameters of these atmospheric hydrodynamic shocks are used as initial condition for another hydrodynamic model developed for acousticwave propagation in the solar interior (Zharkov, 2013, Mon. Not Roy. Astron. Soc. 431, 3414). The model reveals that the depth of energy and momentum deposition by the atmospheric shocks strongly affects propagation velocity of the acoustic wave-packet in the interior. The locations of the first bounces from the photosphere of acoustic waves generated in the vicinity of a flare are seen as ripples on the solar surface, or sunquakes. Mixed proton-dominated beams are found to produce a strong supersonic shock at depths $200-300 \mathrm{~km}$ under the level of the quiet-Sun photosphere, thus, producing well observable acoustic waves; while electron-dominated beams create a slightly supersonic shock propagating down to $5000 \mathrm{~km}$ under the photosphere. This shock can only generate acoustic
\end{abstract}

\footnotetext{
$\triangle$ V.V. Zharkova

valentina.zharkova@northumbria.ac.uk

$\triangle$ S.I. Zharkov

s.zharkov@hull.ac.uk

1 Department of Mathematics and Information Systems, Northumbria University, Newcastle upon Tyne, NE2 1XE, UK

2 Department of Physics and Mathematics, University of Hull, Kingston upon Hull, HU6 7RX, UK
} 
waves at the top layers beneath the photosphere since the shock velocity very quickly drops below the local sound speed. The distance $\Delta$ of the first bounce of the generated acoustic waves is discussed in relation to the minimal phase velocities of wave packets defined by the acoustic cut-off frequency and the parameters of atmospheric shock termination beneath the photosphere.

Keywords: Flares, Relation to Magnetic Field, Magnetic Fields; Acceleration; Flares, Energetic Particles; Heating, in Flares; Helioseismology, Theory

\section{Introduction}

During the past decade it became well established that flares can significantly impact the solar interior, as first predicted by Wolff (1972). The first observation of this impact with the Michelson Doppler Imager (MDI) aboard of the Solar and Heliospheric Observatory (SOHO) seen as ripples travelling from a flare location called a 'sun-quake' was reported by (Kosovichev and Zharkova, 1998) during the flare of 9 July 1996. The original explanation for sunquakes came from the suggestion that these ripples are reflections from the solar surface of acoustic waves induced during flares by some sharp deposition of the momentum (and energy) (Kosovichev and Zharkova, 1995).

The most likely agents delivering such the momentum are considered to be hydrodynamic shocks, which arise in the chromosphere as a result of pressure transients driven by a hydrodynamic response of the ambient plasma to the precipitation of energetic electrons into the chromosphere (Kosovichev and Zharkova, 1995, 1998; Donea and Lindsey, 2005; Kosovichev, 2006). Numerous hydrodynamic simulations have shown that the injection of energetic-particle beams leads to fast heating of the ambient plasma in the corona and chromosphere and its pushing towards the photosphere levels (Somov, Spektor, and Syrovatskii, 1981; Fisher, Canfield, and McClymont, 1985c). This process causes either mild (Allred et al., 2005) or explosive evaporation into the corona (Somov, Spektor, and Syrovatskii, 1981; Fisher, Canfield, and McClymont, 1985c; Zharkova and Zharkov, 2007) of the swept chromospheric plasma, depending on the intensity of the electron beams, at the same time producing low-temperature hydrodynamic shocks moving towards the photosphere and lasting up to a couple of minutes (Zharkova and Zharkov, 2007).

However, the first sunquake (Kosovichev and Zharkova, 1998) indicated essential discrepancies in both timing and energy of the deposited disturbances (momentum) recorded from observation of the sunquake and those delivered by a shock derived from the standard model of a flaring atmosphere heated by electron beams. This provided an additional stimulus to investigate the physics of solar flares and the redistribution of energy from the primary energy release site to the lower atmospheric levels and the solar interior. In turn, it led to the introduction of protons as the agents causing hydrodynamic shocks with the required momentum and travel time (Zharkova and Zharkov, 2007). The authors detected 11 locations showing evidence of hydrodynamic shocks in dopplergrams of the flare of 28 October 2003, which had termination downward velocities from 
1.0 to $2.1 \mathrm{~km} \mathrm{~s}^{-1}$ each lasting up to a couple of minutes. However, only three of them having the termination downward velocities above $1.5 \mathrm{~km} \mathrm{~s}^{-1}$ produced detectable sunquakes on the solar surface (Zharkova and Zharkov, 2007).

Since the first identification of a sunquake by time-distance methods, the development of the helioseismic holography method led to the identification of many more seismic sources during flares of class $\mathrm{M}$ and $\mathrm{X}$ observed with SOHO/MDI and the Solar Dynamic Observatory (SDO) instruments, with a total number growing towards a hundred (see, for example Donea et al., 2006; Zharkova and Zharkov, 2007; Martínez-Oliveros, Moradi, and Donea, 2008; Zharkova, 2008; Matthews, Zharkov, and Zharkova, 2011; Zharkov et al., 2011; Donea, 2011; Alvarado-Gómez et al., 2012; Martínez Oliveros et al., 2012). An increasing number of sunquakes combining observations in different ranges of the electromagnetic spectrum, from high energy hard X-ray (HXR) and $\gamma$-ray emission to lower energy optical and infrared radiation, provides unique opportunities to study in detail the excitation of acoustic oscillations induced by flares. This will lead to a better understanding of various physical conditions in flares and the role of different agents, which are capable causing sunquakes.

In many cases the occurrence of sunquakes is accompanied by strong highenergy emission in HXR, $\gamma$-rays, extreme ultra-violet (EUV), which some authors suggest as the alternative source of pressure transients causing sunquakes. This aspect is related to backwarming of the photosphere by the enhanced coronal and chromospheric radiation leading to white-light and seismic emission (Lindsey and Braun, 2000; Donea, Braun, and Lindsey, 1999; Donea and Lindsey, 2005). Some sunquakes are found in the locations with little or no HXR and white-light emission (Matthews, Zharkov, and Zharkova, 2011; Zharkov et al., 2011), which indicates that, at least, for some flares backwarming heating is not essential.

The shocks formed by a hydrodynamic response of the ambient plasma to precipitation of electron or proton beams seem to be good candidates for the formation of seismic ridges associated with solar flares because they carry sufficient mass and momentum to the solar photosphere (Kosovichev and Zharkova, 1998; Zharkova and Zharkov, 2007). But the question remains as to how exactly these shocks deposit their energy into the solar interior (depths, timescale) and how they can be accounted for from a detailed comparison with seismic observations. These are closely related to the other implications of particle precipitation into a flaring loop, like the formation of magnetic transients and non-thermal plasma ionization leading to prolonged white light emission from solar flares (Zharkova, 2008).

In addition, there are essential changes to the photospheric magnetic field often recorded during a flare well above the noise levels, on both short timescales of minutes (i.e. the duration of the impulsive phase) and longer timescales of hours (before and after a flare) (Kosovichev and Zharkova, 1999, 2001; Zharkova et al., 2005; Sudol and Harvey, 2005; Petrie and Sudol, 2010; Petrie, 2012, 2013). The magnetic field changes in flare locations show magnitudes of 50-120 Gauss that are well above the background noise of any instruments used, as was confirmed by the investigation of magnetic changes, in general, and variations of a magnetic inversion line, in particular (Zharkova et al., 2005). 
The first type of changes, transient (or V-type) changes to the line of sight (LOS) magnetic field, occur in the small pixel-type locations of loop footpoints during the impulsive phase of flares (Kosovichev and Zharkova, 2001; Zharkova et al., 2005), and thus appear closely related to the initial energy release (Zharkova et al., 2011b), often being close in time and location with the appearance of enhanced WL continuum and HXR emission. While the second type, the steptype changes, of LOS magnetic field occur over larger areas including a magnetic inversion line (MIL), or polarity inversion line (PIL) (Kosovichev and Zharkova, 2001; Zharkova et al., 2005; Petrie and Sudol, 2010). These changes appear just before the impulsive phase and continue for the whole duration of flares (a timescale of one or two hours) (Zharkova et al., 2005).

The second type of changes are shown to account for the whole energy deposited during a flare (Kosovichev and Zharkova, 2001; Zharkova et al., 2005; Petrie and Sudol, 2010) and are likely associated with magnetic reconnection producing the flare. While the V-type magnetic field changes, or magnetic transients. can appear in small areas of flares associated with strong HXR emission as a result of the changes in the magnetic field due to precipitation of particle beams (Zharkova and Kosovichev, 2002; Zharkova et al., 2005). These beams carry a strong electric field (Zharkova and Gordovskyy, 2006; Siversky and Zharkova, 2009b), which, in turn, induces an electromagnetic field in the ambient plasma (van den Oord, 1990). This modifies the resulting magnetic field of the loop where the particles precipitate inducing the magnetic field seen as a transient magnetic field (Zharkova et al., 2011b).

Sunquake locations often coincide spatially with one of these magnetic field changes (Zharkova, 2008; Donea, 2011). This led some researchers to suggest that the magnetic changes can directly induce magneto-acoustic waves in the solar interior seen on the solar surface as sunquakes (Hudson, Fisher, and Welsch, 2008; Fisher et al., 2012). However, a close investigation of magnetic field changes in some recent flares did not provide confirmation of a direct association between sunquakes with step-type changes of the magnetic field (Martínez-Oliveros and Donea, 2009; Matthews, Zharkov, and Zharkova, 2011; Zharkov et al., 2011).

Similar to Zharkova (2008), Lindsey and Donea (2008) highlighted that, in the case of magnetic changes, it is the transient component that is the most relevant to acoustic emission. These changes are shown to occur on a timescale of $\tau \approx 2 H / c$ or less, where $H$ is the density scale height and $c$ is the sound speed. In the photosphere, this timescale is about 40s. The question of whether these short-term transient changes during the impulsive phase can be considered as a genuine change in the photospheric magnetic field is still a matter for debate. These localized sign reversals, or 'magnetic anomalies' (e.g. Qiu et al., 2002) are often attributed to the changes in a line profile occurring as the result of the sudden heating of the ambient plasma or the direct bombardment by high energy particles.

However, in the case of the Ni $6768 \AA$ line used by the instruments of the Global Oscillation Network Group (GONG) and MDI aboard the SOHO satellite to make magnetic measurements, the non-LTE simulations have shown that thermal heating occurring at the start of the impulsive phase is insufficient to turn this line into emission when the transient magnetic changes are recorded. 
Instead, it can be caused by a large increase of non-thermal excitation and ionization caused by precipitating electrons, i.e. intense particle bombardment (Zharkova and Kosovichev, 2002).

Observations by Qiu and Gary (2003), which find a good correspondence between the HXR sources and magnetic anomalies in solar flares, rpovided additional support to the hypothesis that magnetic field changes are associated with energetic particles. In addition, the simulations of GONG and MDI observations by Edelman et al. (2004) also concluded that magnetic measurements are less sensitive to the changes in the line profile than Doppler measurements.

Similar conclusions can be drawn for the iron line Fe I 6173 Åused for measuring the magnetic field in the Helioseismic and Magnetic Imager (HMI) instrument onboard the Solar Dynamic Observatory (SDO) satellite following the comparative investigation of this line by the HMI instrument team and the $\mathrm{Ni}$ line used for SOHO/MDI (Norton et al., 2006) (http://hmi.stanford.edu/doc/ Tech_Notes/HMITN-2003-002Line_Choice/line6173_6768_V1.4.pdf).

Further investigation is required into the processes of energy transfer from the magnetic field, reconnected during solar flares, to energetic particles and by these particles to the flaring atmospheres. In this article we investigate the role of mixed beams (electrons and protons) in the heating of the ambient plasma and formation of hydrodynamic shocks, which can cause sunquakes. In Section 2 we discuss the hydrodynamics of the ambient plasma, in Section 3 we probe the shock parameters with the conditions required for detection of ripples on the solar surface by the theory of acoustic-wave propagation in the solar interior and in Section 4 we draw conclusions.

\section{Plasma Response to the Injection of High-Energy Particles}

\subsection{Magnetic Field Changes and Energetic Particles}

\subsubsection{Magnetic Reconnection and Particle Acceleration}

The step-type changes occurring during flares in a LOS magnetic field are likely to reflect the process of 3D magnetic reconnection, the primary energy release mechanisminitiating flares and leading to a change of the magnetic field directivity and/or shear seen in the abrupt changes of a LOS magnitude (Somov, 2000; Priest and Forbes, 2002; Petrie and Sudol, 2010; Petrie, 2012, 2013; Wang et al., 2012).

These changes occur over a large area of the whole active region including a magnetic inversion line (MIL) separating the LOS magnetic field with opposite polarity signs. The MILs were consistently derived from the magnetograms using the Gaussian gradient (Zharkova et al., 2005) in the vicinity of a few different flares, e.g. 14 July 2000 or 23 July 2002 (Kosovichev and Zharkova, 1999, 2001; Zharkova et al., 2005). Zharkova et al. (2005) have also shown that the steptype temporal variations of the magnetic field correlate rather closely with the variations of HXR emission measured during the flare of 23 July 2002. Although, the step-type magnetic field changes occurred only at the locations of some HXR 
sources, while in the others only the transient changes were registered in the MDI magnetograms(Kosovichev and Zharkova, 2001; Zharkova et al., 2005; MartínezOliveros and Donea, 2009; Matthews, Zharkov, and Zharkova, 2011); these are also clearly seen in some of the recent HMI observations by Petrie and Sudol (2010); Petrie (2012).

The difference in magnetic field magnitude between the step positions is found to account for the total energy deposited during a flare (Zharkova et al., 2005). In fact, the estimations show that the magnetic energy calculated from the difference of step-type changes can be two or three times higher than the total energy of HXR, soft X-ray and EUV emissions added together (Holman et al., 2003; Zharkova et al., 2005; Petrie, 2012, 2013). In this sense, all the energy deposited during a flare comes from a magnetic reconnection process, or Lorentz force (of step-type changes), as suggested by Zharkova et al. (2005); Hudson, Fisher, and Welsch (2008). These step-type changes were recenly measured for different flares by Petrie and Sudol (2010); Petrie (2012, 2013); Wang et al. (2012) and quantified with the MHD model (Fisher et al., 2012). The latter simulations are in line with the previous $2 \mathrm{D}$ and $3 \mathrm{D}$ simulations of magnetic reconnection carried out by various authors (see for example books Somov, 2000; Priest and Forbes, 2002, and references therein).

Part of the energy released by magnetic reconnection is converted into the energy of energetic particles. Particle acceleration occurs in current sheets at the intersection of interacting loops, in a diffusive region, where magnetic field lines reconnect. Particles gain their energy from a reconnection electric field directed along the guiding field (Litvinenko, 1996; Zharkova and Gordovskyy, 2004; Zharkova and Agapitov, 2009) while travelling through a 3D magnetic field of a current sheet. During their passage particles are shown to gain subrelativistic energies with power law distributions (Zharkova and Gordovskyy, 2005; Zharkova and Agapitov, 2009). Hence, the particle energy gains are strongly affected by the magnetic field topology in the reconnecting current sheet, e.g. the longer time particles spend inside a current sheet the higher energy they gain (Zharkova et al., 2011a).

Particles with opposite charges, protons and electrons, are simultaneously accelerated as they are dragged from the neutral ambient plasma into a diffusion region during the process of magnetic reconnection. The particles undergo a complex energisation process at the current sheet midplane until they gain sufficient energy to escape from a given 3D magnetic topology. Electrons are accelerated very quickly (within $10^{-6} \mathrm{~s}$ ), so they can break quickly from a current sheet and precipitate into a loop leg. Proton acceleration is slower by three orders of magnitude (about $10^{-3} \mathrm{~s}$ ) leading to protons to gyrate much longer than electrons about the midplane where they gain energy before being eventually ejected. It occurs that very often protons are ejected into a loop leg with the polarity opposite to the leg where electrons are ejected (Zharkova and Gordovskyy, 2004).

In the magnetic topologies with weaker guiding fields accelerated electrons cannot fully break from a current sheet. Instead, they keep returning to its midplane creating electron clouds, which are likely to be seen as coronal sources (Zharkova and Siversky, 2011). This happens because the positive charge from 
protons, which undergo a much longer acceleration gyrating about the midplane, creates an electrostatic electric field, which returns electrons back to the diffusion region. Only after the protons gain sufficient energy to break from the current sheet, they become ejected from this current sheet together with the electrons. Hence, in this case the ejection would contain mixed beams of protons and electrons in each leg. The ratio of proton-to-electron numbers is shown to be dependent on the magnetic field topology in the current sheet (Zharkova and Gordovskyy, 2004; Zharkova and Agapitov, 2009).

Moreover, a full kinetic particle-in-cell (PIC) approach for current sheets in the corona (Siversky and Zharkova, 2009a) or in the heliosphere (the heliospheric current sheet, HCS) (Zharkova and Khabarova, 2012) indicate that full separation of the particles are the rare events applicable for the topologies with large magnitudes of the guiding field. For many other magnetic field topologies this separation is only partial. In the other words, there can be a larger number of electrons than protons in one loop leg and a larger number of protons than electrons in the other leg.

Evidently, in the corona it is rather difficult to distinguish signatures of accelerated particles from the acceleration site from those modified during their precipitation into loop footpoints (or loop legs). While in the HCS it is possible to obtain the direct in-situ measurements of accelerated particle parameters. These measurements showe a close agreement of the observed parameters (ion and electron density, velocity profiles, pitch angle distributions) of solar wind particles crossing the sector boundary of the HCS with those derived from the PIC simulations carried out for low-density current sheets using a 3D magnetic topology similar to the HCS (Zharkova and Khabarova, 2012). These direct insitu measurements confirming the PIC simulation results in the HCS magnetic field topology lend a strong support to the results derived from PIC simulations for current sheet conditions of the solar corona suitable for flares (Siversky and Zharkova, 2009a).

During reconnection particles of the ambient plasma pass through a current sheet and gain subrelativistic energies and negative power-law energy distributions with spectral indices being essentially different for electrons and protons/ions. Hence, there are four particle populations that appear during a magnetic reconnection process (Gordovskyy et al., 2005): 1) power-law sub-relativistic electrons, 2) power-law subrelativistic protons (produced by direct acceleration in a current sheet) combined with 3) quasi-thermal (up to 10-20 keV) electrons, and 4) quasi-thermal protons (produced by Alfven and other waves in the current sheet exhausts). The third and fourth populations have energies much higher than the thermal energies gained from a magnetic reconnection process (Somov, 2000; Gordovskyy et al., 2005). All four types of particles gain subrelativistic velocities from a magnetic field reconnection. Together with high velocities, these particles also gain high momenta (Gordovskyy et al., 2005; Zharkova and Zharkov, 2007), which they deposit into the footpoints of a flaring loop.

Thus, it is evident that magnetic reconnection energy is delivered to flaring atmospheres by mixed electron and proton beams, which most likely become only partially separated at the current sheet they pass and injected into the loop legs. In addition, these mixed beams can be accompanied by quasi-thermal 
protons or electrons appearing because of Alfvn waves generated at the current sheet exhausts.

As an example, there can be $70 \%$ electrons and $30 \%$ protons (in particle numbers) ejected into one leg and $70 \%$ protons and $30 \%$ electrons into the other one. The protons in the first leg have much lower velocities than the electrons and their energy is not high enough for them to be detected in $\gamma$-ray emission. While in the second leg the electrons have gained lower energies, and their number is rather small, so they produce weak HXR emission. The charge neutralisation process will include ambipolar diffusion in both cases, and not only a formation of return currents from the ambient plasma and lower energy electrons in the beam, as in the case of pure electron beams (Zharkova and Gordovskyy, 2006).

\subsubsection{Transient Changes of Magnetic Field}

Let us now evaluate the appearance of transient magnetic fields induced by energetic electron beams precipitating into footpoints of the flaring atmospheres. These particles deposit their energy by collisions and Ohmic heating of the ambient particles causing a hydrodynamic response of the ambient plasma that we will discuss in Section 2.2.

It is evident that beams of electrons should carry a strong electrostatic electric field sometimes exceeding the local Dreicer field by the two orders of magnitude (Zharkova and Gordovskyy, 2006). This electric field strongly affects the spectral indices of HXR bremsstrahlung emission in solar flares (Siversky and Zharkova, 2009a). At the same time, these beam electrons are turned back to the corona by their own electrostatic electric field.

This happens at stopping depths of the electrons with a lower cutoff energy where they start travelling across the magnetic field of a loop (Siversky and Zharkova, 2009a). Then, following Faraday's law, these electrons can induce a strong magnetic field in the ambient plasma, which we have called in the Introduction a transient magnetic field (van den Oord, 1990). This field can exist as long as the beam electrons are injected into the atmosphere as shown by the time-dependent Fokker-Planck simulations (Siversky and Zharkova, 2009a). This transient magnetic field is exactly what is observed in many flares (Sudol and Harvey, 2005; Zharkova et al., 2005; Petrie and Sudol, 2010) indicating the precipitation of electron beams.

From the difference in photon spectral indices at higher and lower energies one can derive the induced electric field as $\Delta E_{b} \simeq(1-40) \times E_{D}$, where $E_{D}$ is a local Dreicer field (Zharkova and Gordovskyy, 2006). Then, from Faraday's law: $\frac{1}{c} \frac{\partial \vec{B}}{\partial t}=-\nabla \times \vec{E}_{b}$, where $c$ is the speed of light. Hence, in order to produce an induced magnetic field, the electrons need to move across the existing magnetic field. This is easily achievable in the case of returning electrons with a lower cutoff energy, which move across the loop at the point where they are turned back to the source by their own electrostatic electric field, as shown by Fokker-Planck numerical simulations (Siversky and Zharkova, 2009b).

This induced transient magnetic field is of opposite polarity to that originally present in a given footpoint. The magnitude of this transient magnetic field can be in the range of $30-120 \mathrm{G}$ as measured from the magnetic field variations 
in flare locations (Kosovichev and Zharkova, 2001; Zharkova et al., 2005). The energy released in this process is linked to the energy of energetic particles, heating the ambient plasma, which will be discussed in Section 2.2 .

\subsubsection{The Radiative Processes and Backwarming Heating}

Since energetic particles produce high-energy HXR bremsstrahlung and $\gamma$-ray emission, these emissions can play some role in the additional heating of lower atmospheric levels (called backwarming heating) leading to white-light flares (Hudson, 1972; Machado, Emslie, and Avrett, 1989; Metcalf et al., 2003; Donea et al., 2006; Zharkova, 2008), which often accompany seismic emission in flares. A substantial fraction of the continuum emission of white-light flares coming from the overheated upper atmosphere that can result in a radiative backwarming of the photosphere by recombination radiation (Hudson, 1972; Machado, Emslie, and Avrett, 1989), which was recently simulated using radiative hydrodynamics by Allred et al. (2005).

The photosphere is assumed to absorb the part of coronal and chromospheric radiation that is emitted downward. Therefore, it becomes heated not only by collisions of beam electrons or protons with the ambient particles but also by the HXR and ultra-violate (UV) or EUV radiation coming from the upper layers. The immediate effect of this absorption in the visible spectrum is mostly dissociation of neutral hydrogen leading to white-light emission caused by long-lasting recombination of free electrons into neutral hydrogen atoms and creation of $H^{-}$ ions defining the photospheric opacity. This process lasts until the conditions reach the pre-heated level after which the heating is stopped. The radiation energy estimated from white-light flares is found to be close to that measured using seismic emission (Donea et al., 2006). This allowed Donea et al. (2006) to suggest backwarming as an additional source of delivering energy to the photosphere and leading to the acoustic emission closely associated with the continuum emission.

However, Allred et al. (2005) have pointed out that in their hydrodynamic models considering heating by electron beams of a very moderate power, the backwarming heating is not very effective during the impulsive phase when the hydrogen emission is formed mostly by non-thermal ionization by beam electrons (Zharkova and Kobylinskii, 1993). Moreover, it was recently shown that the location of white-light emission in a limb flare is very close to the location of low energy $(<100 \mathrm{keV})$ HXR emission (Martínez Oliveros et al., 2012) that significantly reduces the likelihood that this WL emission will affect the flare seismic response, which occurs at much deeper atmospheric levels.

After beam heating is stopped (say, in 10-30 seconds), the thermo-conductivity continues to heat the lower atmosphere further, thus, increasing its emission in all lines and continua leading to further increases of the line and continuum opacity that is reported by Zharkova and Kobylinskii (1993) and confirmed by Allred et al. (2005). Moreover, Allred et al. (2005) reported that any additional heating of the ambient plasma does not increase the backwarming heating of the photosphere, which remains gentle since it is governed by a high opacity of the continuum radiation. 
On the other hand, Zharkova (2008) pointed out that the hydrogen ionization degree is increased by up to six orders of the magnitude by collisions with beam electrons approaching nearly full ionization level while the kinetic temperature of hydrogen atoms remains as low as it should be at the photosphere. The increased ionization caused by collisions with beam electrons produces strong emission in hydrogen lines and continua (Zharkova and Kobylinskii, 1993; Zharkova, 2008). This means that the opacity of continuum radiation is increased that, it turn, keeps this radiation locked in the atmosphere for a timescale comparable with the difference (reaching sometimes tens of minutes) between the impact excitation rates of hydrogen atoms by beam electrons and the recombination rates of the ambient electrons with these hydrogen atoms, as discussed by Zharkova (2008).

Hence, the presence of white-light emission does not necessarily mean that there is backwarming heating of the plasma, but can be just a sign of overionization of the ambient plasma by subrelativistic particles (electrons and protons) (Zharkova, 2008).

\subsubsection{Delivered Momenta and Timing of Seismic Signatures}

The detailed evaluation of the momenta required to produce the sunquakes observed in the flare of 28 October 2003 (Zharkova and Zharkov, 2007) has shown that during the event there are 11 shocks detected in the Dopplergram datacubes taken around the flare location. The shocks lasted 1.5-2.5 minutes each with the downward velocities ranging between 1.0 and $2.1 \mathrm{~km} / \mathrm{s}$. However, ripples associated with sunquakes were not detected in all the locations of these downward motions (Zharkova and Zharkov, 2007) but only in the three locations where the downward velocities of the shocks exceeded $1.5 \mathrm{~km} \mathrm{~s}^{-1}$. This means that only a certain amount of momentum delivered to the photosphere within a very short timescale can initiate visible sunquakes.

Despite of the occurrence of seismic signatures in flares often being close to the locations of HXR and WL emission (Donea, 2011), they do not always coincide with them as reported for the flare of 28 October 2003 (Zharkova and Zharkov, 2007). A similar picture was detected with the GONG data for the flare of 14 December 2006 (Matthews, Zharkov, and Zharkova, 2011) where only two seismic sources were associated with HXR sources, while the other two did not show any or very little HXR emission. The first sunquake of Cycle 24 that occurred on 15 February 2011 revealed no significant HXR emission associated with one of the sunquake locations, while the other showed some HXR emission, which was much lower than that measured during the main flaring event.

From this point of view, even if backwarming does exist, which is often the case, its starting time after the impulsive phase and extended duration (for up 40 minutes) cannot deliver energy and momentum fast enough to the photosphere. For this reason, it cannot account for the timing and energy required to produce seismic ripples close to the start of HXR emission (Kosovichev and Zharkova, 1998; Zharkova and Kosovichev, 2000, 2002) or within a minute or two after the HXR onset (Zharkova and Zharkov, 2007).

The points discussed above increase the requirements for delivering energy and momentum to the photosphere and lead to consideration of hydrodynamic shocks 
produced not only by electron (Kosovichev and Zharkova, 1998; Kosovichev, 2007) but also by proton beams (Zharkova and Zharkov, 2007) or magnetohydrodynamic shocks produced by Lorentz force (Fisher et al., 2012). These cases emphasize the importance of considering mixed beams of electrons and protons, which are most likely occur during the flare onset, as discussed in Section 2.1.1.

\subsection{Hydrodynamic Response}

In order to emulate the dynamics of a flaring atmosphere, let us simulate the hydrodynamic response of the two-temperature ambient plasma to the injection of energetic particles (either electrons or protons) considering the ion viscosity (Somov, Spektor, and Syrovatskii, 1981). We solve the two energy (for electrons and protons), continuity and momentum conservation equations with the ion viscosity term (Somov, Spektor, and Syrovatskii, 1981; Zharkova and Zharkov, 2007), including the radiative cooling by optically thin coronal emission and optically thick hydrogen emission (Kobylinskii and Zharkova, 1996). The initial conditions at the time $\mathrm{t}=0$ are considered to be the photospheric ones: $T\left(\xi_{0}, 0\right)=$ $6700^{\circ} \mathrm{K}, n\left(\xi_{0}, 0\right)=10^{13} \mathrm{~cm}^{-3}, v\left(\xi_{0}, 0\right)=0 \mathrm{~cm} \mathrm{~s}^{-1}$, and the original atmosphere is considered to be the simple exponential one.

This approach is essentially different from the simplified one-temperature approach developed by Fisher (1989) without consideration of the ion viscosity. The hydrodynamic conditions in that approach were applied to a pre-heated semiempirical atmosphere using quiet-Sun physical parameters (Vernazza, Avrett, and Loeser, 1981). The outcome of such the hydrodynamic simulation was then used by Fisher et al. (2012) for the explanation of some sunquake properties. The differences in the results of these two different models will be discussed in the next sections.

\subsubsection{Heating Functions by Energetic Particles}

Zharkova and Zharkov (2007) evaluated the ambient heating caused by electron, proton beams or their mixture, and the implications for the ambient plasma responding to this heating (variations of temperature, density and macrovelocities); we will use the similar methodology in this article. Let us briefly recapture the main points.

Similar to Somov, Spektor, and Syrovatskii (1981); Zharkova and Zharkov (2007), for the heating function by beam electrons we use the formula derived from a continuity equation by Syrovatskii and Shmeleva (1972). This heating function is essentially different from the heating function used by Nagai and Emslie (1984); Allred et al. (2005); Fisher (1989). Their heating function was derived by Emslie $(1978,1980)$ from the flux conservation equation, where the maximum energy deposition by electrons occurs much higher in the chromosphere compared to that derived by Syrovatskii and Shmeleva (1972). Furthermore, the flux conservation approach was shown by ? to produce a strong singularity in the electron heating function (in fact, it becomes infinity) at the stopping depth of low-energy electrons. In order to avoid this singularity, Emslie $(1978,1980)$ selected the shape of the heating function with the maximum heating occurring above this stopping depth, which is not the best approximation as shown by ?. 
In this article, similarly to Zharkova and Zharkov (2007), the heating of the ambient plasma by electron beams occurs due to Coloumb collisions and Ohmic heating by precipitating particles (electrons, protons and their mixture). The heating by proton and/or mixed beams is considered via Coulomb collisions and generation of kinetic Alfvn waves (KAWs) with their subsequent dissipation in Cherenkov resonance at the atmospheric depths where their velocities are higher than the local Alfvn ones (Gordovskyy et al., 2005).

As expected, the heating by electron or proton/mixed beams with power law energy distributions is found (Zharkova and Zharkov, 2007) to be strongly dependent on the initial beam parameters: softer and weaker beams deposit their energy mainly in the corona and upper chromosphere whilst harder and more powerful beams deposit more energy deeper in the lower chromosphere. The other heating mechanism for electron beams, Ohmic dissipation in a self-induced electric field, contributes significantly to the heating of the plasma at coronal levels but it does not significantly affect the heating of the lower atmosphere (Zharkova and Gordovskyy, 2006; Siversky and Zharkova, 2009b).

Evidently, before returning to the source in the corona, some of lower energy electrons lose the bulk of their energy in Coulomb collisions contributing to heating of the upper layers in the flaring atmosphere and depositing momentum into lower atmospheric levels (Zharkova and Zharkov, 2007). At the same time, these electrons create their own strong electrostatic electric field. This electric field leads to a large number of lower energy electrons returning back to the corona, or moving nearly across the magnetic field at some depth in the chromosphere (electrical stopping depth). This is caused by the significant reduction in their energy by self-induced electric field and anisotropic scattering in the presence of this field (Siversky and Zharkova, 2009b). This process would definitely increase the energy deposited at higher atmospheric levels and reduce the amount of energy deposited at lower atmospheric levels via a hydrodynamic response.

\subsubsection{Hydrodynamic Response of a Flaring Atmosphere}

In this article, we are mainly interested in hydrodynamic shocks, as the sources of sunquakes, produced in the ambient plasma heated by energetic particles. Let us calculate hydrodynamic responses and resulting shocks produced by different mixtures of particles, electrons and protons, injected into the loop legs from the top (see Section 2.1.1).

The physical conditions in a flaring atmosphere are described by a plasma density $n$, electron $T_{e}$ and proton/ion $T_{i}$ temperatures, and a vertical velocity $v$ as a function of a vertical coordinate $z$, or a column density $\xi$, and time $t$. The initial conditions at the time $\mathrm{t}=0$ are considered to be the photospheric ones: $T\left(\xi_{0}, 0\right)=6700^{\circ} \mathrm{K}, n\left(\xi_{0}, 0\right)=10^{13} \mathrm{~cm}^{-3}, v\left(\xi_{0}, 0\right)=0 \mathrm{~cm} \mathrm{~s}^{-1}$. Then we calculate these parameters for different times as a hydrodynamic response of the 1D solar atmosphere to the injection of electrons and/or protons followed by radiative cooling using the continuity, momentum, and energy equations for the ambient electrons and protons/ions following Zharkova and Zharkov (2007)(see also Somov, Spektor, and Syrovatskii, 1981; Fisher, Canfield, and McClymont, 1985a,b,d; Allred et al., 2005; Fisher, 1989). 
Similar to Somov, Spektor, and Syrovatskii (1981), in our simulations we consider the momentum equation for a two- temperature plasma approach, in addition to the two energy (for electrons and for ions) equations and continuity equation for injected particles (Somov, Spektor, and Syrovatskii, 1981; Zharkova and Zharkov, 2007). This allows us to include the momentum deposited by these particles that is shown to be very important for particle dynamics (Brown and Craig, 1984). Fisher (1989) also includes the momentum equation in a very simplified form without considering the energy exchange between the ambient electrons and ions included in our analysis (Zharkova and Zharkov, 2007). For the radiative cooling term we consider the losses from optically thin emission of all elements with the coronal abundance, similar to Fisher (1989) and the emission in optically thick hydrogen lines and continuum calculated for the 5 plus continuum model of the hydrogen atom (Kobylinskii and Zharkova, 1996).

As a result of the hydrodynamic simulation for a flaring atmosphere, one can derive (within the first seconds after the beam injection onset) a sharp increase of the temperature and decrease of the density in the corona, formation of a transition region just above the chromosphere and the formation of a low temperature condensation in the lower chromosphere (Somov, Spektor, and Syrovatskii, 1981; Fisher, Canfield, and McClymont, 1985a,b,d; Zharkova and Zharkov, 2007). These processes are accompanied by the bulk plasma motion: the fast explosive upward motion of the photospheric plasma into the chromosphere and corona, called a 'explosive evaporation' and supersonic motion of the low-temperature condensation towards the photosphere.

These two motions of the ambient plasma (upwards and downwards) are reported in all the hydrodynamic simulations and the velocities of this bulk motion are called macrovelocities (Somov, Spektor, and Syrovatskii, 1981; Nagai and Emslie, 1984; Fisher, Canfield, and McClymont, 1985a,b,d). These macromotions are widely investigated from the blue and red-shifted spectral measurements in UV and $H \alpha$ emission, respectively (Zarro, Slater, and Freeland, 1988). For some events, or some beam parameters, the momenta of these two motions can be nearly equal (Zarro et al., 1988). While some other events reveal only the upward motions with blue shifts indicating explosive evaporation without noticeable downward motions (to be seen as red shifts). For some other events there are stronger red shifts observed and smaller blue ones, indicating a more gentle chromospheric evaporation.

The hydrodynamic heating of flaring atmospheres caused by injected electron and proton beams has been already discussed in great detail in our previous papers (Zharkova and Zharkov, 2007; Zharkova, 2008). Zharkova and Zharkov (2007) simulated and presented the full set of plots for electron temperature, ambient density and macrovelocity in response to the injection of electron and proton beams. They showed that the low temperature condensation formed in response to either of these beams has a temperature below $10000 \mathrm{~K}$ and the density exceeding $10^{14}-10^{15} \mathrm{~cm}^{3}$. This condensation moves downward to the photosphere with a speed from $10^{7} \mathrm{~cm} \mathrm{~s}^{-1}$ in the chromosphere slowing down to a few units of $10^{5} \mathrm{~cm} \mathrm{~s}^{-1}$ before its full termination at the photosphere. Furthermore, Zharkova and Zharkov (2007) showed in their Figure 9 that the temporal profile of the termination of the shock produced by protons was very 
close to the temporal profiles directly measured from Dopplergrams for the flare of 28 October 2003.

In this article we decided to explore the heating and hydrodynamic response in flaring atmospheres extended towards the photosphere and beneath in an attempt to detect the times when or if the lower-temperature shocks can reach the photosphere and how deep they can push their photospheric level into the solar interior compared to this level in the quiet atmosphere.

In Figure 1 the upward and downward macrovelocities of the bulk of the ambient plasma are plotted versus a column depth similar to the plot presented by Zharkova and Zharkov (2007) reflecting the macromotion of the ambient plasma in response to the injection of a powerful electron beam with an initial energy flux of $F_{0}=5 \cdot 10^{11} \mathrm{erg} \cdot \mathrm{cm}^{-2} \mathrm{~s}^{-1}$ and spectral index $\gamma=4.9$ (top plot) and mixed beam ( $80 \%$ of electrons and $20 \%$ of protons) with the initial energy flux of $F_{0}=3 \cdot 10^{12} \mathrm{erg} \cdot \mathrm{cm}^{-2} \mathrm{~s}^{-1}$ and spectral index $\gamma=3$ (bottom plot).

From Figure 1 it can be seen that there is a very significant difference in the generation of macrovelocities by different agents, which define different velocities for chromospheric evaporation into the corona as well as different depths for the formation of low- temperature shocks and their termination velocities. The macrovelocity variations caused by a pure electron beam with the initial flux of $2 \times 10^{12} \mathrm{erg} \cdot \mathrm{cm}^{-2} \mathrm{~s}^{-1}$, and spectral index $\approx 5$ (top plot) reveal rather high velocities of chromospheric evaporation (up to $10^{8} \mathrm{~cm} / \mathrm{s}$ ) occurring from the upper chromosphere (column depth up to $5 \times 10^{19} \mathrm{~cm}^{-2}$ ). In addition, there is also the lower temperature shock formed immediately below this depth, reaching velocities of up to $10^{7} \mathrm{~cm} \mathrm{~s}^{-1}$ in the upper chromosphere while terminating at the column depth just before $10^{21} \mathrm{~cm}^{-2}$ with a velocity of up to $(1-2) \cdot 10^{5} \mathrm{~cm} \mathrm{~s}^{-1}$. This reflects the fact that pure electron beams have maximum heating function in the upper chromosphere (Syrovatskii and Shmeleva, 1972; Somov, Spektor, and Syrovatskii, 1981), thus forming the shock directly under the transition region.

For the beams with $20 \%$ protons and $80 \%$ electrons the initial energy flux is noticeably increased (see Figure 1, bottom plot) leading to a shift of the energy deposition maximum from the lower corona to the upper chromosphere. This reduces the macrovelocties of chromospheric evaporation into the corona from $10^{8} \mathrm{~cm} \mathrm{~s}^{-1}$ in the first ten seconds to a factor of $10^{5} \mathrm{~cm} \mathrm{~s}^{-1}$ after 130 seconds. At the same time, the lower temperature shock is now split into two shocks. One is moving from the lower corona towards the chromospheric column depth of $10^{19} \mathrm{~cm}^{-2}$ terminating at the depths of $10^{20} \mathrm{~cm}^{-2}$ that is a chromospheric level. The second shock appears at the column depth $4 \times 10^{20} \mathrm{~cm}^{-2}$ and terminates at the depth $1 \times 10^{21} \mathrm{~cm}^{-2}$. The maximum velocity approaches $10^{6} \mathrm{~cm} \mathrm{~s}^{-1}$ in the first shock and a few units of $10^{5} \mathrm{~cm} \mathrm{~s}^{-1}$ in the second one.

The macrovelocities of the bulk of the ambient plasma induced by a hydrodynamic response to the injection of mixed beams are plotted in Figure 2 for the electron-dominated beam with $\mathrm{F}=5 \times 10^{12} \mathrm{erg} \cdot \mathrm{cm}^{-} \mathrm{s}^{-1}$ and in Figure 3 for the proton-dominated beam with the initial energy fluxes $\mathrm{F}=5 \times 10^{13} \mathrm{erg} \cdot \mathrm{cm}^{-2} \mathrm{~s}^{-1}$.

It can be observed that a further increase of the initial energy flux and momentum (Figure 2) for a slightly more intense mixed beam with an initial flux of $5 \times 10^{12} \mathrm{ergcm}^{-} \mathrm{s}^{-1}$ (containing $70 \%$ of electrons and $30 \%$ of protons) 


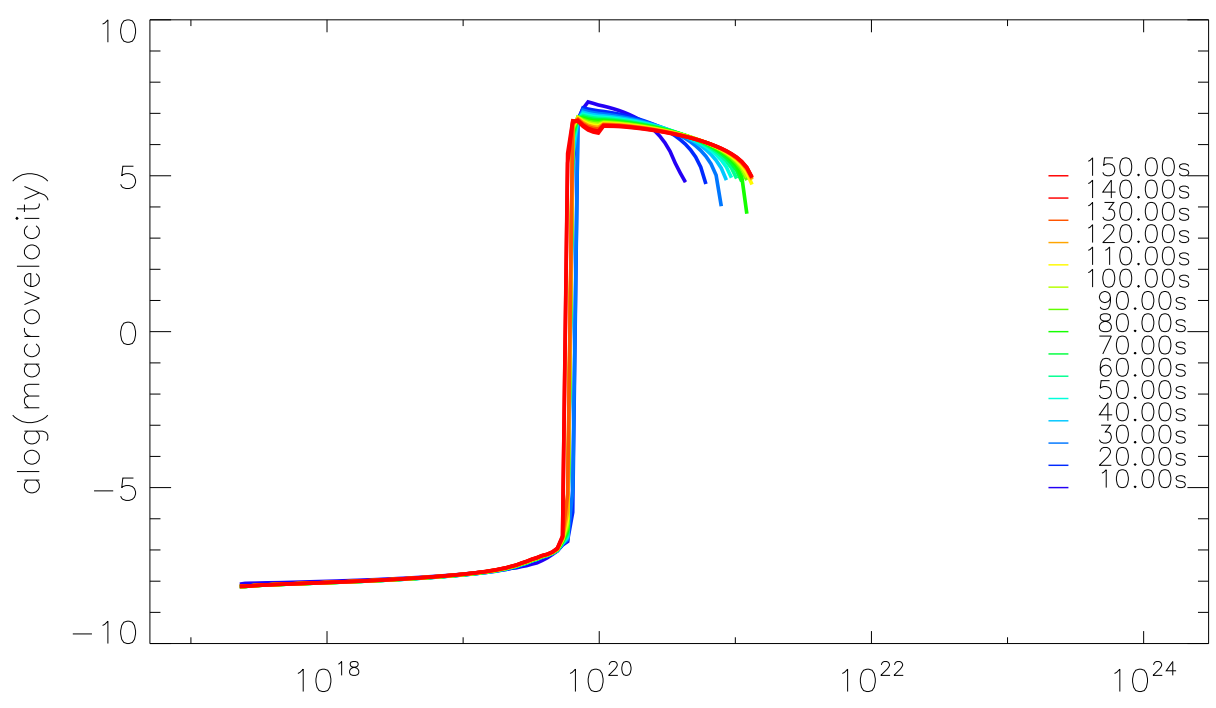

Column depth, $\xi, \mathrm{cm}^{-2}$

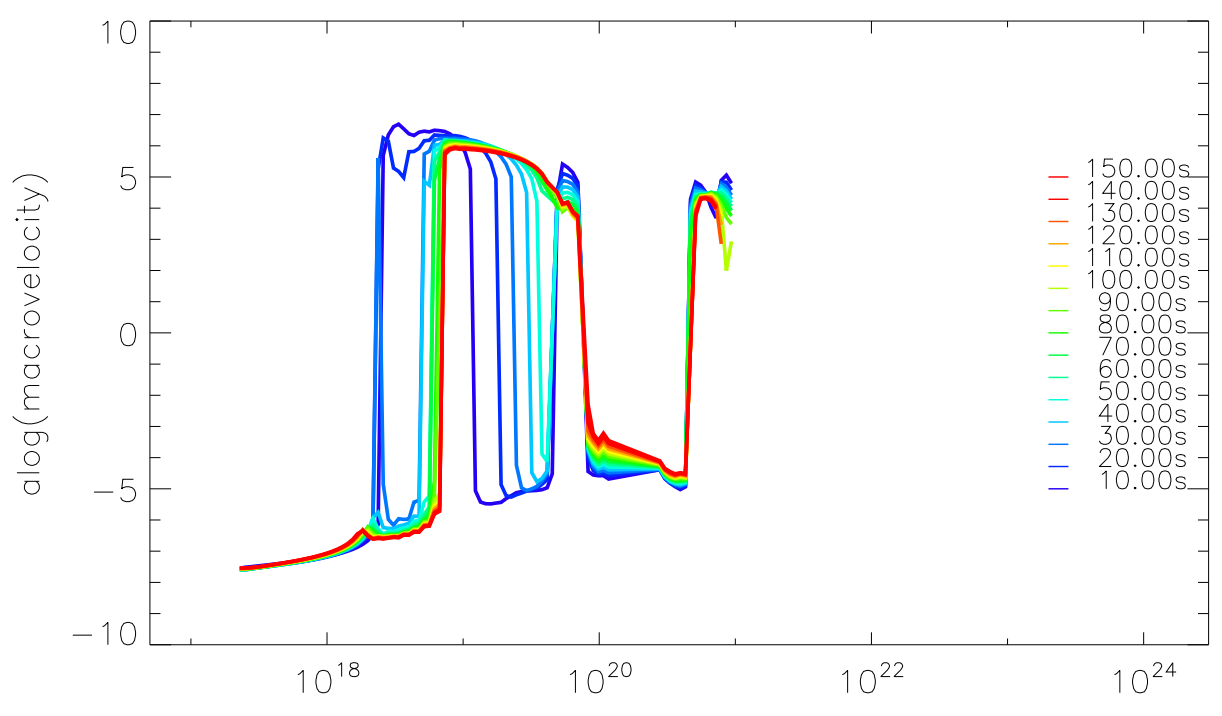

Column depth, $\xi, \mathrm{cm}^{-2}$

Figure 1. Snapshots of the macrovelocity profiles for different times after the beam injection onset (colour-marked description on the right) in function of the column depth (X-axis) with the shocks formed at different times after the injection of: top plot - a pure electron beam with the parameters derived from HXR emission (the initial energy flux $5 \cdot 10^{11} \mathrm{erg} \cdot \mathrm{cm}^{-2} \mathrm{~s}^{-1}$, spectral index $\gamma=4.9$ ), bottom plot - a mixture of $80 \%$ of an electron beam and $20 \%$ of a proton beam (particle number) (an initial energy flux $3 \times 10^{12} \mathrm{erg} \cdot \mathrm{cm}^{-2} \mathrm{~s}^{-1}, \gamma=3.0$ ). The macrovelocity profiles are given on $\mathrm{Y}$ axis in logarithmic scale in $\mathrm{cm} s^{-1}$. 


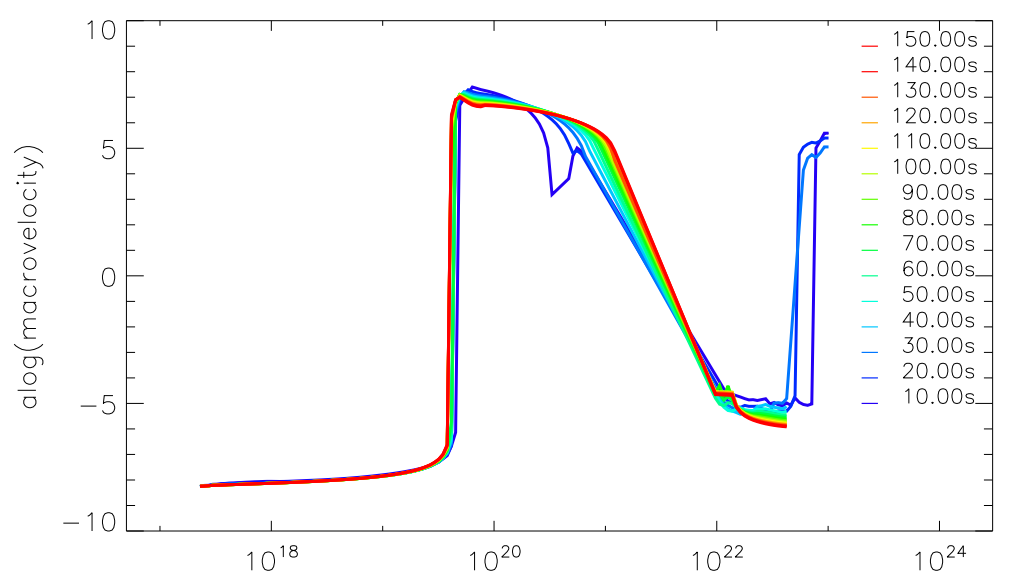

Column depth, $\xi, \mathrm{cm}^{-2}$

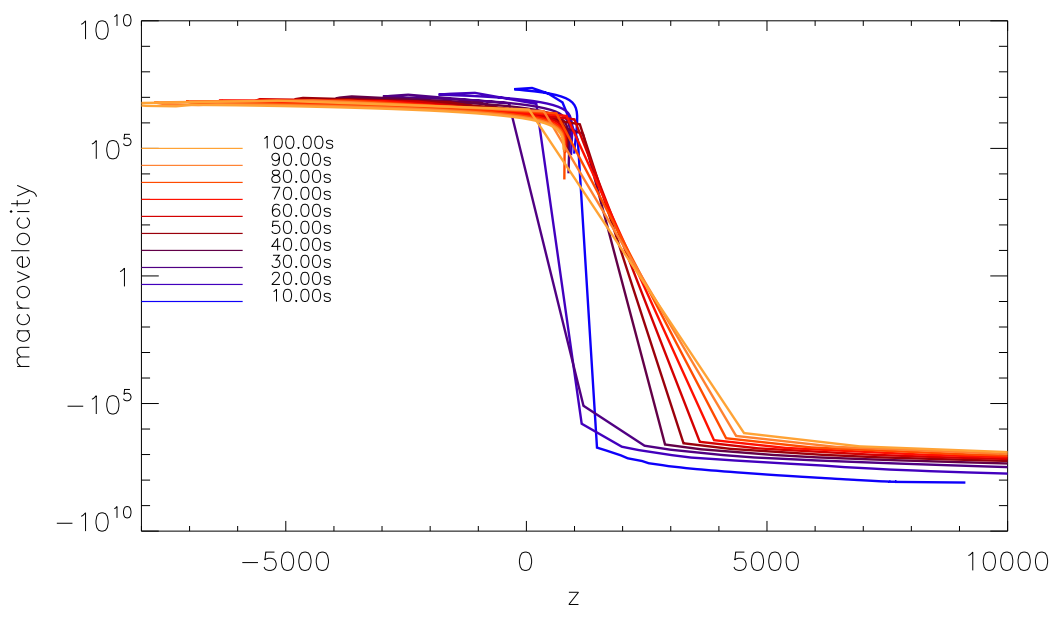

Linear depth, $k m$

Figure 2. Snapshots of the macrovelocity profiles (Y-axis, $\mathrm{cm} \mathrm{s}^{-1}$ ) for different times after the beam injection onset (colour-marked description in the plot insets) in function of the column depth $\xi$ (the top plot) and the linear depth Z (the bottom plot) with respect to the quiet-Sun photosphere (zero level). The shocks are formed in response to the injection of a beam with $70 \%$ of electrons and $30 \%$ of protons with the initial energy flux of $\mathrm{F}=5 \times 10^{12}$ $\mathrm{erg} \cdot \mathrm{cm}^{-2} \mathrm{~s}^{-1}, \gamma=3.0$ Note that the pre-flare photospheric level is at the column depth of $10^{23} \mathrm{~cm}^{-2}$ (or linear depth is zero) while during a flare this level moves under the level of the quiet photosphere by $5000 \mathrm{~km}$. 


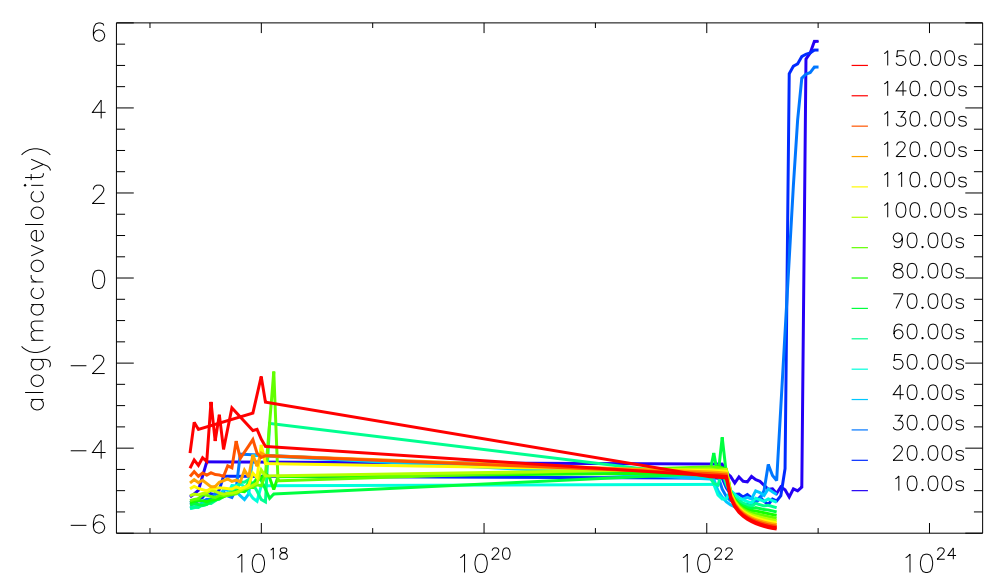

Column depth, $\xi, \mathrm{cm}^{-2}$

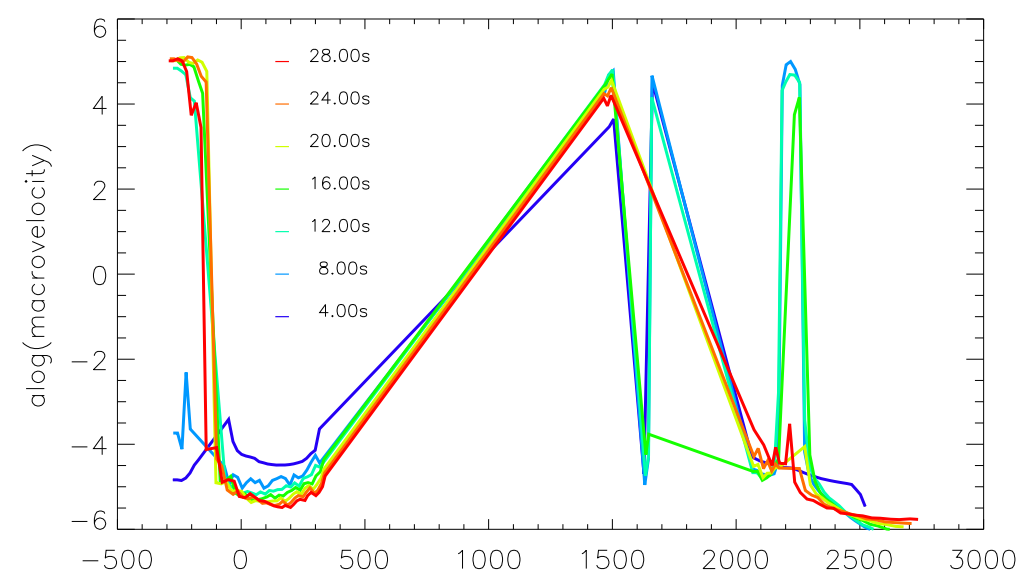

Linear depth Z, $\mathrm{km}$

Figure 3. Snapshots of the macrovelocity profiles (Y-axis, logarithmic scale, in units of $\mathrm{cm}$ $s^{-1}$ ) versus column depth $\xi$ (top plot) and linear depth $\mathrm{Z}$ (bottom plot) with the hydrodynamic shocks formed at different times after the injection of the beam with $70 \%$ of protons and $30 \%$ of electrons with an initial energy flux of $\mathrm{F}=5 \times 10^{13} \mathrm{erg} \cdot \mathrm{cm}^{-2} \mathrm{~s}^{-1}, \gamma=5.0$. The ordinates in both the plots show the logarithms of the absolute values of the macrovelocity with a positive sign corresponding to plasma moving towards the solar interior and a negative sign to plasma moving to the corona. Note that the pre-flare photospheric level is located at the column depth of $10^{23} \mathrm{~cm}^{-2}$ (or linear depth is zero), while during a flare this level moves under the level of the quiet photosphere by $500 \mathrm{~km}$. 
leads to formation of the double shocks, similar to the ones described in Fig. 1 (bottom plot). For a larger contribution of protons, the upper shock becomes spread much wider from column depth of $8 \times 10^{19} \mathrm{~cm}^{-2}$ to $10^{22} \mathrm{~cm}^{-2}$ with the second shock starting at the depth of $5 \times 10^{22} \mathrm{~cm}^{-2}$ and terminating at the depth $10^{23} \mathrm{~cm}^{-2}$ with the velocity of a few units $10^{5} \mathrm{~cm} / \mathrm{s}$.

The upper shocks can produce Moreton waves in the chromosphere, which are occasionally observed in association with sunquakes (Kosovichev and Zharkova, 1998; Warmuth and Mann, 2011), while the bottom shocks can be considered as the strong candidates for producing sunquake ripples (Zharkova and Zharkov, 2007).

The simulation plotted in Figure 3 in function of column depth (top plot) considering the beams of protons ( $70 \%$ of a total abundance) and electrons (30\% of a total abundance) shows a reduction of the velocities of chromospheric evaporation (up to $10^{6} \mathrm{~cm} \mathrm{~s}^{-1}$ ) while still keeping the wide first low-temperature shock spreading from the whole chromosphere with the second low-temperature shock starting at a depth of $5 \times 10^{22} \mathrm{~cm}^{-2}$ and terminating at the depth $10^{23}$ $\mathrm{cm}^{-2}$ with the velocity of a few units of $10^{5} \mathrm{~cm} \mathrm{~s}^{-1}$.

To show the linear depth of the formation of this second shock, we plot in Figures 2 and 3 the macrovelocities of the ambient plasma versus a column depth (top plots) and a linear depth (bottom plots) with the zero level corresponding to the undisturbed photospheric level (defined at the start of simulations by the boundary condition for the hydrodynamic equations).

In the case of the mixed beam dominated by electrons (see Figure 2) the second shock can travel from the photosphere and beneath to a distance of about $5000 \mathrm{~km}$ (bottom plot). Since the two shocks are initiated in the upper and middle chromosphere of a flaring atmosphere, the latter is swept under the quiet-Sun photospheric level. This leads to large linear depths (up $5000 \mathrm{~km}$ ) for these shocks to travel beneath the quiet-Sun photosphere, before their full termination. In fact, the shocks are both created and terminated smoothly at increasingly deeper levels approaching the depth of $5000 \mathrm{~km}$.

While with a stronger contribution of protons in hydrodynamic heating, the second shock generated by this mixed beam is terminated in the solar interior at depths of about $300 \mathrm{~km}$ below the quiet-Sun photosphere (see Figure 3, bottom plot). This happens because the proton-dominated beam creates the shock in a much denser plasma at the bottom of the chromosphere (Zharkova and Zharkov, 2007). As a result, this shock sweeps much denser plasma under the quiet photospheric level, and it travels a much shorter linear distance before terminating. In this particular simulation, the shock moves only $300 \mathrm{~km}$ beneath the quiet photosphere level, before it terminates as shown in Figure 3 (bottom plot).

The results of the hydrodynamic simulations, in general, confirm, within the limits of the simplified model used (Fisher, 1989), the estimations by Fisher et al. (2012) of the momentum and energy delivered by hydrodynamic shocks. However, our hydrodynamic results have a big advantage in having the initial condition for the quiet photosphere (before flare onset) and consideration of the two-temperature plasma with the energy exchange between the ambient electrons and protons (see Section 2.2). That allows us to make a reliable calculation 
of a linear depth within a flaring atmosphere compared to the quiet atmosphere level.

While in the model by Fisher (1989); Fisher et al. (2012) the initial condition is prescribed to the semi-empirical quiet-Sun model derived from fitting the observed solar emission in multiple lines (Vernazza, Avrett, and Loeser, 1981), which is dependent on settings of the non-LTE model and is known to underestimate the height of the quiet photosphere level (Somov, Spektor, and Syrovatskii, 1981). This immediately moves the zero level of a flaring atmosphere to a level, which is beneath the photosphere in the models used by Somov, Spektor, and Syrovatskii (1981); Zharkova and Zharkov (2007).

In addition, the mild heating functions by electron beams with the initial fluxes below $10^{11} \mathrm{erg} \cdot \mathrm{cm}^{-2} \mathrm{~s}^{-1}$ used in the model by Fisher (1989); Fisher et al. (2012) do not produce the hydrodynamic responses with the shocks formed in the lower chromosphere and the photosphere like those reported in this article. These numeric limitations impose some restriction on the outcomes in the hydrodynamic models used by Fisher (1989); Fisher et al. (2012) allowing only detection of weak shocks in the chromosphere above the photospheric level, which are unable to produce seismic signatures in flares similar to the ones reported in this study.

The occurrence of either single or double hydrodynamic shocks and their termination either in the solar atmosphere or interior well under the undisturbed photospheric level can explain the observational features related to seismic responses, or sunquakes, occurring in the solar interior and their occasional links with Moreton waves occurring in the chromosphere and coronal waves measured by the SOHO Extreme ultraviolet Imaging Telescope (EIT) (Warmuth and Mann, 2011). Only mixed beams (with a substantial proportion of protons) carrying large energies and momenta can form two shocks: one in the upper atmospheric levels leading to Moreton waves, and the other one at the photospheric level, which terminates deeply in the solar interior leading to sunquakes (see Section 3 below).

\subsubsection{The Momentum and Energy Delivered by a Hydrodynamic Shock}

The momentum delivered by a hydrodynamic shock can be evaluated using the simple formula (Zharkova and Zharkov, 2007):

$$
P_{h d}=\Sigma_{t} m v_{v e r t}(t)
$$

where the summation is done over the time from 0 to $\tau, \tau$ is the duration of the impact causing the seismic waves, $m$ is the mass of the plasma delivering the momentum related to the flaring area $A$ where the momentum is deposited, $V_{\text {vert }}$ is a starting velocity of the plasma at the impact time and $t$ is the duration of the impact.

For the known plasma mass density $\rho=m_{H} \cdot n$ where $n$ is the particle density per volume defined from hydrodynamic solutions, this equation can be rewritten as follows: 
Table 1. Estimations of momenta $P_{h d}$ and kinetic energies $E_{k}$ of hydrodynamic shocks in the three seismic sources terminated to areas $A$ with downward (vertical) velocities $V_{\text {vert }}$, with duration $T$ of the sunquake ripples, and the sunquake average horizontal velocity, $V_{\text {horiz }}$ (see for details the article by Zharkova and Zharkov (2007))

\begin{tabular}{lrccccc}
\hline Sources & $\begin{array}{r}\text { area, } A, \\
\mathrm{~cm}^{2}\end{array}$ & $\begin{array}{r}V_{\text {vert }}, \\
\mathrm{cm} \mathrm{s} \mathrm{s}^{-1}\end{array}$ & $\begin{array}{c}\tau, \\
\mathrm{s}\end{array}$ & $\begin{array}{c}V_{\text {horiz }}, \\
\mathrm{cm} \mathrm{s} \mathrm{s}^{-1}\end{array}$ & $\begin{array}{c}P_{h d}, \\
\mathrm{~g} \cdot \mathrm{cm} \cdot \mathrm{s}^{-1}\end{array}$ & $\begin{array}{c}E_{k} \\
\mathrm{erg}\end{array}$ \\
\hline$S 1$ & $5.05 \times 10^{17}$ & $2.15 \times 10^{5}$ & 90 & $40.8 \times 10^{5}$ & $4.2 \times 10^{22}$ & $4.2 \times 10^{29}$ \\
$S 2$ & $3.34 \times 10^{17}$ & $2.00 \times 10^{5}$ & 70 & $38.0 \times 10^{5}$ & $4.0 \times 10^{22}$ & $4.0 \times 10^{29}$ \\
$S 3$ & $2.22 \times 10^{17}$ & $1.75 \times 10^{5}$ & 70 & $35.4 \times 10^{5}$ & $3.7 \times 10^{22}$ & $3.5 \times 10^{29}$ \\
\hline
\end{tabular}

$$
P_{h d}=\Sigma_{t} m v_{\text {vert }}(t) \approx \rho \cdot A \cdot v_{v e r t}^{2} \cdot \tau,
$$

where $\rho$ is the average density of the plasma delivering the momentum, $A$ is the flaring area where the momentum is deposited, $v_{v e r t}$ is the averaged vertical velocity of the plasma propagation at the impact time and $\tau$ is the duration time of the impact.

Then, the kinetic energy delivered by such the hydrodynamic shock can be written as:

$$
E_{k}=\Sigma_{t} m v(t) \cdot v_{\text {vert }}(t) / 2 \approx 1 / 2 \rho \cdot A \cdot v_{\text {vert }}^{3} \cdot \tau,
$$

Of course, this energy is deposited over a depth (column depth $\xi$ or linear depth $z$ ) of a flaring atmosphere, into which this shock moves during time $t$. Only a small part of the shock is deposited into acoustic waves; this is fraction, which is left at the moment when the hydrodynamic shock is terminated into dense plasma causing the acoustic waves in the solar interior.

In order to estimate this fraction of the energy, one needs to estimate the plasma density, a flare area, where the sunquake occurrs, duration of shock propagation (or existence of sunquake), and a measured Doppler velocity of the downward motion (hydrodynamic shock) in the location of the sunquake. Then, the momentum and energy deposited by a shock in a flare with the given physical conditions can be estimated following the techniques described step-by-step in the earlier paper by Zharkova and Zharkov (2007). An example of the estimation of such the momentum for the sunquakes detected for the flare of 28 October 2003 (Zharkova and Zharkov, 2007) is summarised in Table 1.

It has to be noted that the energy deposited by the hydrodynamic shocks generated in a flaring atmosphere presented in Table 1 is much higher than that derived from the acoustic waves produced by flares, which normally ranges in $10^{27}-10^{28}$ erg (Donea, 2011; Zharkov et al., 2013). This is because in the sunquakes, first, researchers measure only the energy of the first bounce of acoustic waves induced by these shocks derived as a ridge in the time-distance 
diagrams while there can be many more bounces occurring beyond the datacube considered. Second, some of the deposited energy can be spent on a drag force inside the solar interior, and, third, the properties of the photosphere (density, temperature, etc.) where the acoustic waves reflected from are also unknown. All these properties need yet to be investigated in more detail from observations of sunquakes.

\section{Hydrodynamic Response of the Solar Interior and Formation of Acoustic Waves}

Sunquakes are relatively rare events taking place in active regions during flares, when a flare deposits sufficient momentum and energy into the lower atmosphere and, under certain conditions, can generate acoustic waves in the solar interior. These waves are seen in photospheric Dopplergrams as circular shaped surface ripples accelerating away from the source region where the impact occurs. However, due to random background oscillations, such ripples are often difficult to distinguish.

Therefore, helioseismic methods such as time-distance diagram analysis (Kosovichev and Zharkova, 1998) and helioseismic holography (see for example Lindsey and Braun, 1997; Donea, Braun, and Lindsey, 1999, and references therein) are usually applied for sunquake detection from the suitably processed series of the 2D line-of-sight velocity observations (dopplergrams), called datacubes (of the size varying from $180 \mathrm{Mm}$ x $180 \mathrm{Mm}$ to $250 \mathrm{Mm}$ x $250 \mathrm{Mm}$ )), obtained with 45 seconds -1 minute cadence for the duration of two or more hours.

The time-distance diagram technique is a direct observational method using a position of the quake source in the $3 \mathrm{D}$ datacube $(2$ horizontal dimensions and time) to remap it to a $2 \mathrm{D}$ time-distance image while deriving the surface velocity variations as a function of time and radial distance from the assumed source. Sunquake ripples in this wave are seen as a ridge on the time-distance diagram (2D image).

On the other hand, the helioseismic egression measurements are based on the theoretical modelling of acoustic signals propagating from a point source and emanating outwards from the flare location. This allows one to produce 2D egression power maps of the acoustic sources and sinks, where the quake signatures are represented by compact bright kernels of the enhanced emission surrounded by the acoustically absorbing interior (Lindsey and Braun, 1997). This procedure was used in many flares (e.g. see Donea et al., 2006; Zharkov, Zharkova, and Matthews, 2011; Matthews, Zharkov, and Zharkova, 2011; Zharkov et al., 2011).

Both helioseismic methods show the very localized nature of the origin point of seismic sources (where the ripples start from) validating the point source assumption used in the theoretical interpretation (Kosovichev and Zharkova, 1995; Zharkov, 2013) and in the observational analysis of Dopplergrams for detecting ripples (see, for example Kosovichev and Zharkova, 1998; Zharkov, Zharkova, and Matthews, 2011; Matthews, Zharkov, and Zharkova, 2011; Zharkov et al., 2011). 

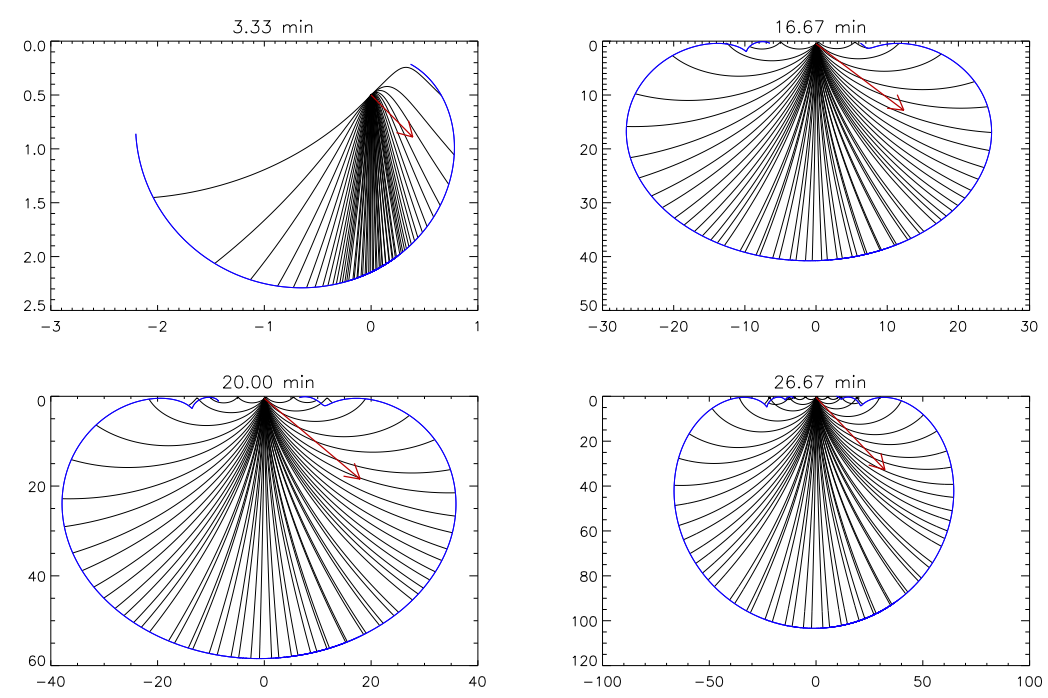

Figure 4. The individual acoustic rays (generated at the depth of $500 \mathrm{~km}$ and travelling to the bottom of the plot) by a moving supersonic source depositing a momentum below the photosphere (the origin) under some angle to the local vertical for the times shown above the plots. The photosphere is denoted by the top line, the $\mathrm{X}$-axis denotes a distance in $\mathrm{Mm}$ from the point of momentum deposition. The red arrow shows the direction of a momentum deposition. Note a reflection of the rays from the photosphere level at the time of 20 minutes (the bottom right plot), which can be observed as a ripple on the surface, or a sunquake, generated about the central point of the momentum deposition.

In this paper we consider that these point-like sources are the hydrodynamic shocks of the ambient plasma formed in flaring atmospheres by the mixed beams, which deposit their energy and momentum into the production of acoustic wavepackets in the solar interior. In fact, if the sources are the hydrodynamic shocks caused by mixed beams as shown in Figures 2 and 3, then it becomes evident that these shocks move under the level of the quiet-Sun photosphere for a distance from a few hundred to a few thousand kilometers. With every second these shocks move downwards with a supersonic speed (up to $12 \mathrm{~km} \mathrm{~s}^{-1}$ for a mixed beam with a larger fraction of protons or up to $6-8 \mathrm{~km} \mathrm{~s} s^{-1}$ for electrondominated mixed beam, with the adiabatic soundspeed, $c$ being around $7 \mathrm{~km}$ $s^{-1}$ near the surface and growing to around $20 \mathrm{~km} \mathrm{~s} s^{-1}$ at $5000 \mathrm{~km}$ depth.

These hydrodynamic shocks from the atmosphere above can be used as the initial condition for another hydrodynamic model developed for acoustic wave propagation in the solar interior. As the initial hydrodynamic shock terminates within relatively shallow depths and with strongly supersonic velocities, the generated waves are formed at the point of deposition as a closed cone around the velocity vector in the solar interior, which, in accordance to Fermat principle, propagates deeper into the interior refracting due to the increasing temperature and reflecting back to the photosphere (see Figure 4, bottom plots).

The model of the interior can be assumed to be either a polytrope (Zharkov, 2013) or the standard interior model (Christensen-Dalsgaard et al., 1996) used in this study. By solving the hydrodynamic equations for acoustic wave propa- 
gation in the interior (Zharkov, 2013), one can evaluate parameters of the generated acoustic wave packets and the condition of their detection from Doppler observations.

We note that the wave packet comprises a large number of individual rays with different wavelengths/frequencies). Then, as it was shown by Zharkov (2013) the vertical shock perturbation moving with a supersonic velocity can generate the set of multiple acoustic waves, from which only the waves with the phase speed exceeding a certain threshold (Zharkov, 2013, see Equation 5.8) can produce the observable acoustic waves.

An individual ray characterized by a constant (along the ray) frequency, $\omega$, and horizontal wavenumber, $k_{h}$, initialized at given depth, will have two, upper and lower, turning points (see Figure 1 in Zharkov, 2013). The first upper turning point along the ray defines its first surface appearance (as a first ripple), the lower turning point indicates where the wave changes its direction of its motion in the interior by being reflected back to the surface. Then the propagating ripples correspond to a sequence of the source-generated acoustic rays from the packet reaching in succession their upper turning points.

The source of the deposited impulse, depending on its properties, generates a family of the rays that provides the solution to the ray equations in the phase space and defines the generated wave front. As the source is located in the interior, the first ray (out of all generated by the source) to reach its upper turning point defines the minimal distance where the ripple is formed. This distance will depend on the source depositing a momentum, a depth where this momentum is deposited and the interior model as described below.

For a near-surface source, i.e. for the ray initiated near its upper turning point, the first surface appearance, or the minimal distance, can be approximated by the ray's skip distance, $\Delta$, the distance between its surface bounces. This distance depends on the ray's horizontal phase speed, $\omega / k_{h}$ (see, for example Christensen-Dalsgaard et al., 1996; Zharkov, 2013). For the polytrope model of the solar interior, used in Zharkov (2013), the minimal skip distance, $\Delta$, or the distance from the point of the initial impulse deposition to the first ripple occurrence, is derived from equations (A1) in the Appendix A1 (Zharkov, 2013) as follows:

$$
\Delta\left(k_{h}, \omega\right)=(\omega)^{2} \pi m /\left(k_{h}\right)^{2} g=\left(V_{p h}\right)^{2} \pi m / g,
$$

where $\mathrm{g}$ is the gravitational constant, $g=2.67 \times 10^{-4} \mathrm{Mm} \mathrm{s}^{-2}$, $\mathrm{m}$ is the polytrope index, $V_{p h}=w / k_{h}$ is the horizontal speed of wave propagation.

For the supersonic (non-oscillatory) source (see Section 5.3 in Zharkov, 2013, and the corresponding plots), the waves of the packet are generated in a cone/sphere around the velocity vector, with the ray frequencies increasing with the angle measured from the velocity vector (see Equation (5.5) in Zharkov (2013)). In this case, the observations of high-frequency waves will be also limited by the Nyquist frequency and cadence of the series $(8.33 \mathrm{mHz}$ for MDI, $11.11 \mathrm{mHz}$ for $\mathrm{SDO} / \mathrm{HMI}$ ). Thus, if the observations are made at a certain Nyquist frequency, $\omega_{N}$, the high-frequency waves may not be observed, so further restrictions for observable ripples are considered, namely $\omega \leq \omega_{N}$ holds. 
This leads to the threshold condition for the minimal phase speed, $v_{p h}^{\min }$, defining the condition for registering the first ripples on the surface by the following relation (see Equation (5.8) in Zharkov (2013)):

$$
v_{p h}^{\min }=\frac{v c}{\sqrt{\left(1-\frac{\omega_{\alpha c}^{2}}{\omega_{N}^{2}}\right) v^{2}-c^{2}}}
$$

where $\omega_{\alpha c}$ is the acoustic cutoff frequency and c is the speed of sound.

The propagation of these waves is tracked by the rays, shown in Figure 4. The abscissa defines the horizontal distance in Mm about the locations of supersonic disturbances (shocks) and ordinate shows depth, $z_{s}$, under the photosphere. The moving source shown in Figure 4 introduces an anisotropy in the produced acoustic wave packet. The waves become generated in a cone around the velocity vector, with the frequencies increasing with the increase of the angle between the initial shock velocity vector and the ray take-off direction.

The phase speed of ripple appearance increases with the distance from the source of the original impact as seen in all the time-distance diagrams of sunquakes (see, for example Kosovichev and Zharkova, 1998; Zharkov et al., 2011). Since the propagation of surface ripples from a near-surface source can be determined by the phase speed (Zharkov, 2013), the minimal distance, $\Delta$, where these ripples are observed can be estimated from Equation (4) after a substitution of the minimal phase speed given in Equation (5). For the horizontal velocities from 15 to $45 \mathrm{~km} / \mathrm{s}$ reported by Kosovichev and Zharkova $(1995,1998)$, the first bounce normally comes to the surface at a skip distance about $\Delta=40 \mathrm{Mm}$ that is seen in about 20-25 minutes after the initial impulse deposition in the source.

The other ripples, which are observed further from the source, are, in fact, the first bounces of the other rays in the wave packet. A linear phase speed of rays increases with a descrease of the ray frequency (or an increase of the ray velocity) (see formula (5). This leads to the ripple produced by the next ray to be seen much further from the first one. This process will continue until the location of the next ripple occurrence exceeds the size of the datacube. This defines the conditions, under which the ripples can be detected depending on the initial deposition velocity.

For example, for the initial supersonic shock produced by electron-rich beam and deposited with the velocity of $v\left(z_{s}\right)=10 \mathrm{~km} \mathrm{~s}^{-1}$ in the interior with the sound speed of $c\left(z_{s}\right)=7.5 \mathrm{~km} \mathrm{~s} s^{-1}, \omega_{\alpha c}\left(z_{s}\right) / 2 \pi=5.4 \mathrm{mHz}$ and for $\omega_{N}=$ $2 \pi \cdot 8.3$ (corresponding to the dopplergram cadence), the condition that the cyclic frequency along the ray is no greater than Nyquist frequency $\omega_{N}$, gives us the minimum phase speed estimate, $v_{p h}$, over of $60 \mathrm{~km} \mathrm{~s}^{-1}$. After substituting this speed into formula (4), the minimal skip distance where we can observe the first ripple is found exceeding $80 \mathrm{Mm}$.

It is evident that the ripples moving with the speed from 60 to $80 \mathrm{~km} / \mathrm{s}$ (or average speed of $\left.70 \mathrm{~km} \mathrm{~s}^{-1}\right)$ reach a distance beyond $180 \mathrm{Mm}(70 \cdot 45 \cdot 60=189$ $\mathrm{Mm}$ ) within less than 45 minutes. Hence, this ripple propagation will be rather difficult to detect from the time-distance diagrams as it will be seen far away from the source. This would suggest that for a relaible observation of ripples 
on the solar surface associated with acoustic waves the limit of their horizontal phase velocity should not roughly exceed $50 \mathrm{~km} \mathrm{~s}^{-1}$.

These analytical estimations are in line with the numerical simulations showing generation of acoustic waves by convective vortices in the quiet-Sun interior (Kitiashvili et al., 2011; Moll, Cameron, and Schüssler, 2011) reporting also supersonic movements at the vortices, similar to those derived from the observations of sunquakes (e.g. see Kosovichev and Zharkova, 1998; Kosovichev, 2007; Zharkov, Zharkova, and Matthews, 2011; Matthews, Zharkov, and Zharkova, 2011; Zharkov et al., 2013). These similarities lend a solid support to the analytical theory of acoustic waves induced by the hydrodynamic processes in flares (Zharkov, 2013).

In addition, the geometry of wave propagation will largely depend on the angle, under which the initial shock enters the photosphere, and, because of the rays being generated within a cone, their frequencies can be growing away from the initial velocity vector. In the other words, if at the sunquake locations a supersonic movement is observed with a strong anisotropy in the amplitude in one direction, this points to the initial atmospheric shock to be deposited under some angle to the surface in this direction. Therefore, it is logical to suggest that this mechanism plays an important, if not the key, role in the sunquake wave front generation, which is linked to the parameters of hydrodynamic shocks induced in flaring atmospheres by energetic particles.

In the application to the models considered in this study, the atmospheric hydrodynamic shocks generated by a proton-dominated beam (Fig. 3, bottom plot) are found deposited consistently at a shallow depth range between 200 and $300 \mathrm{~km}$ under the photospheric level. The high supersonic speed of this shock $v>c\left(\mathrm{v}=12 \mathrm{~km} \mathrm{~s}^{-1}\right.$ and $\left.\mathrm{c}=6 \mathrm{~km} \mathrm{~s}^{-1}\right)$, the shock propagation creates a sharp set of waves shown in Figure 4, derived from the hydrodynamic theory about a movement of the supersonic source in the solar interior (see Section 5.3 in Zharkov (2013)).

In the case of the shocks created by electron beams (see Fig. 2, bottom plot), at each instant these shocks are deposited over a large range of depths from 0 to $5000 \mathrm{~km}$ under the quiet sun photosphere. In the case of the shocks generated by electron-dominated beams, the speed of shock $(8 \mathrm{~km} / \mathrm{s})$ only marginally exceeds the sound speed at the top of the interior $(6 \mathrm{~km} / \mathrm{s})$.

Similar to the previous case with a proton-dominated beam, the acoustic waves generated by electron-dominated shocks are located within a cone of the velocity vector with the angular cone width determined by the ratio $v / c$. This means that the shocks still can generate individual rays at the upper depths of the solar interior shown as separate lines in Figure 4. However, because these shocks are terminated smoothly at the deeper interior layers, this would result in the larger upper skip distances (Equation (4) for their ripples to be observed. This means that these shocks can be reflected from the surface at much greater distances than the standard datacube size of 180x180 $\mathrm{Mm}^{2}$ used in helioseismology of sunquakes.

In addition, in deeper layers of the interior the speed of the shocks generated by electron-rich beams drops very quickly below the local sound speed that would elliminate their ability to generate acoustic waves shown in Figure 4. Therefore, 
the summary set of acoustic waves (rays) produced by electron-dominated shock would be rather scattered in the interior. Thus, in this particular occasion the shock cannot not generate significant visible effects on the photosphere seen as ripples within the given datacube sizes.

For the cases presented in this paper we find that the hydrodynamic theory in the solar interior with acoustic cut-off frequency (Zharkov, 2013) can logically explain the generation of the observable acoustic waves, or sunquakes, in solar flares observed in the datacubes of $180 \times 180 \mathrm{Mm}^{2}$. These detectable acoustic waves are shown linked closer to the shocks induced by the mixed protondominated beams. While the acoustic waves induced by the electron-dominated shocks can have much wider spectrum and, thus, can be reflected from the surface at much larger distances than the standard datacubes used in the helioseismology of sunquakes.

Of course, when observers register sunquakes within a given datacube, they can only derive the energy of the first bounce of these acoustic packets, leaving behind the majority of waves, which travel much further in the solar interior. This limits the measurements of acoustic wave energy to approximately $1-10 \%$ of their total energy. In addition, to estimate the fraction of the energy transferred from the hydrodynamic shocks deposited from a flaring atmosphere, presented in Section 2.2.3, to the acoustic waves in the solar interior, discussed in this section, one needs to take into account the drag force of the waves during their propagation in this interior leading to its heating. Also, the properties of the photosphere where the waves are reflected from, while producing enhancement of the plasma above the surface seen as ripples, need also to be further investigated from the observations of sunquakes. These are the important tasks for the future studies before any modelling for one-to-one reproduction of acoustic waves generated during flares can be carried out.

\section{Conclusions}

In this article, we have combined for the first time the two hydrodynamic models associated with solar flares to derive the conditions for favourable observation of acoustic waves, or sunquakes. The first hydrodynamic model is developed for a two-temperature flaring atmosphere heated by mixed particle beams (protons plus electrons) (Somov, Spektor, and Syrovatskii, 1981; Zharkova and Zharkov, 2007). The heating produces an increase of large macromotions of the ambient plasma upwards (explosive evaporation) and downward (low-temperature hydrodynamic shocks) moving to the photosphere and beneath.

The velocities and directivity of these shocks are considered to be the initial condition for the second hydrodynamic model (Zharkov, 2013) used for the investigation of acoustic wave generation and propagation in the solar interior beneath a flare. This model defines the acoustic cut-off frequency and the minimal phase velocity, above which the acoustic waves can be measured from the surface observations.

This approach is different from the one with a single kinetic temperature and simplified momentum equation used by Fisher et al. (2012). Also in this study 
the initial condition used was considered for the undisturbed photosphere with a density of $10^{13} \mathrm{~cm}^{-3}$ and a temperature of $6700^{\circ} \mathrm{K}$ that sets the quiet-Sun photospheric level. While in the other hydrodynamic models the authors consider semi-empirical model of aflaring atmosphere as the initial condition.

The heating functions for beam electrons and protons are derived from the continuity equations (Syrovatskii and Shmeleva, 1972; Somov, Spektor, and Syrovatskii, 1981; Gordovskyy et al., 2005) allowing us to increase their initial energy fluxes of precipitating beams to very high magnitudes (up to $5 \cdot 10^{13}$ $\mathrm{erg} \cdot \mathrm{cm}^{-2} \mathrm{~s}^{-1}$, for a proton-rich beam). This is contrary to the heating function derived from the flux conservation equation used by Fisher et al. (2012), which is known to have the well-known limitations on energy deposited by lower energy electrons at the chromosphere (Mauas and Gómez, 1997). This, in turn, imposes essential limitations on a maximum energy flux of injected beams, which can be used in hydrodynamic models.

These hydrodynamic simulations allowed us to calculate the macrovelocity profiles varying not only with column depth, but also with a linear depth measured from the photospheric level of the quiet Sun. We compared the linear depths of deposition of the hydrodynamic shocks generated by hydrodynamic responses of the ambient plasma to the injection of pure electron beams, electronrich (with some fraction of protons) beams and proton-rich (with some fraction of electrons) beams.

It turned out that the shocks induced by pure electrons are terminated either above the photosphere or just at its surface. The shocks induced by protonreach beams are found terminated at the narrow band of 200-300 km beneath the quiet solar photosphere while the shocks produced by electron-reach beam are terminated smoothly over the depths from 0 down to $5000 \mathrm{~km}$ beneath the quiet-Sun photosphere.

The shocks induced by mixed beams gain the downward macrovelocity at the surface of about $8 \mathrm{~km} \mathrm{~s}^{-1}$ (electron-rich beam) and $12 \mathrm{kms}^{-1}$ (proton-rich beam) exceeding the sound speed of $6 \mathrm{~km} \mathrm{~s}^{-1}$ at the depths just under the photosphere increasing to to $20 \mathrm{~km} \mathrm{~s}^{-1}$ in the deep interior. The shocks are found to terminate at some deeper layers with the speed of a few $\mathrm{km} \mathrm{s}^{-1}$.

By using these hydrodynamic shocks from the above atmosphere as the initial condition for another hydrodynamic model developed for acoustic wave propagation in the solar interior (Zharkov, 2013) following a standard polytrope model, we evaluate the parameters of the generated acoustic wave packets and the conditions of the detection of these waves from Doppler observations.

Indeed, a vertical shock perturbation moving with a supersonic velocity is shown to generate a cone-like multiple acoustic wave-packet in the direction of shock motion. From this wave packet only the waves with the horizontal phase speed exceeding a certain threshold can produce observable acoustic waves, or sunquakes (Zharkov, 2013).

According to this second hydrodynamic model of acoustic wave propagation in the solar interior, the atmospheric shock caused by a proton-rich mixed beam propagates with a high supersonic speed and deposits its momentum at the linear depths of 200-300 km beneath the quiet photospheric level. This means 
this shock generates the set of acoustic waves with very close parameters, which propagate in the solar interior.

While the shock produced by the electron-rich beam considered in this article is deposited nearly continuously over the depth from beneath the surface down to $5000 \mathrm{~km}$. In this case the reflection of acoustic waves from the surface happens at a large distance of hundreds $\mathrm{Mm}$, which are not available in the datacubes used in local helioseismology of flares. Also the continuous deposition over the depth of $5000 \mathrm{~km}$ means that the acoustic waves produced by this shock can smoothly travel in the interior and become smoothly reflected from the photosphere at large distances from the source without producing any noticeable signatures (ripples) on the surface withing the datacube used.

We noted in this study that the energy deposited by hydrodynamic shocks is much higher than that derived from the observations of sunquakes in flares. This reflects the facts that researchers measure from sunquakes so far only the energy of the first bounce of acoustic waves induced by these shocks. This limits the measurements of acoustic wave energy to approximately $1-10 \%$ of their total energy. In addition, some energy is spent on a drag force inside the solar interior, whose properties need yet to be investigated as well as the properties of the photosphere where the waves are reflected from, while producing enhancement of the plasma above the surface seen as ripples.

Therefore, further theoretical investigation is required of the conditions for generation, propagation, and directivity of acoustic waves in the solar interior induced by the supersonic shocks of diferent nature (hydrodynamic or magnetohydrodynamic) generated in flaring atmospheres and deposited beneath the solar surface of the quiet-Sun with different velocities and at different angles.

Acknowledgments The authors would like to express their deepest gratitude to the Leverhulme Trust for supporting the Workshop at Mullar Space Science Laboratory, UCL, in September 2013 for discussion of the key questions of sunquake origins, where this paper was initiated. The authors also wish to thank the anonymous referee for very useful comments from which the paper strongly benefited.

\section{Declaration of Conflict of Interests}

The authors declare that they have no conflicts of interest.

\section{References}

Allred, J.C., Hawley, S.L., Abbett, W.P., Carlsson, M.: 2005, Radiative Hydrodynamic Models of the Optical and Ultraviolet Emission from Solar Flares. Astrophys. J. 630, 573. DOI. ADS.

Alvarado-Gómez, J.D., Buitrago-Casas, J.C., Martínez-Oliveros, J.C., Lindsey, C., Hudson, H., Calvo-Mozo, B.: 2012, Magneto-Acoustic Energetics Study of the Seismically Active Flare of 15 February 2011. Solar Phys. 280, 335. DOI. ADS.

Brown, J.C., Craig, I.J.D.: 1984, The importance of particle beam momentum in beam-heated models of solar flares. Astron. Astrophys. 130, L5. ADS. 
Christensen-Dalsgaard, J., Dappen, W., Ajukov, S.V., Anderson, E.R., Antia, H.M., Basu, S., Baturin, V.A., Berthomieu, G., Chaboyer, B., Chitre, S.M., Cox, A.N., Demarque, P., Donatowicz, J., Dziembowski, W.A., Gabriel, M., Gough, D.O., Guenther, D.B., Guzik, J.A., Harvey, J.W., Hill, F., Houdek, G., Iglesias, C.A., Kosovichev, A.G., Leibacher, J.W., Morel, P., Proffitt, C.R., Provost, J., Reiter, J., Rhodes, E.J. Jr., Rogers, F.J., Roxburgh, I.W., Thompson, M.J., Ulrich, R.K.: 1996, The Current State of Solar Modeling. Science 272, 1286. DOI. ADS.

Donea, A.: 2011, Seismic Transients from Flares in Solar Cycle 23. Space Sci. Rev. 158, 451. DOI. ADS.

Donea, A., Lindsey, C.: 2005, Seismic Emission from the Solar Flares of 2003 October 28 and 29. Astrophys. J. 630, 1168. DOI. ADS.

Donea, A., Braun, D.C., Lindsey, C.: 1999, Seismic Images of a Solar Flare. Astrophys. J. Lett. 513, L143. DOI. ADS.

Donea, A., Besliu-Ionescu, D., Cally, P.S., Lindsey, C., Zharkova, V.V.: 2006, Seismic Emission from A M9.5-Class Solar Flare. Solar Phys. 239, 113. DOI. ADS.

Edelman, F., Hill, F., Howe, R., Komm, R.: 2004, The Effect of Spectral Line Shape Changes on GONG Observations of Oscillations and Flares. In: D. Danesy (ed.) SOHO 14 Helioand Asteroseismology: Towards a Golden Future, ESA Special Publication 559, 416. ADS.

Emslie, A.G.: 1978, The collisional interaction of a beam of charged particles with a hydrogen target of arbitrary ionization level. Astrophys. J. 224, 241. DOI. ADS.

Emslie, A.G.: 1980, The effect of reverse currents on the dynamics of nonthermal electron beams in solar flares and on their emitted X-ray bremsstrahlung. Astrophys. J. 235, 1055. DOI. ADS.

Fisher, G.H.: 1989, Dynamics of flare-driven chromospheric condensations. Astrophys. J. 346, 1019. DOI. ADS.

Fisher, G.H., Canfield, R.C., McClymont, A.N.: 1985a, Flare Loop Radiative Hydrodynamics - Part Seven - Dynamics of the Thick Target Heated Chromosphere. Astrophys. J. 289, 434. DOI. ADS.

Fisher, G.H., Canfield, R.C., McClymont, A.N.: 1985b, Flare Loop Radiative Hydrodynamics Part Six - Chromospheric Evaporation due to Heating by Nonthermal Electrons. Astrophys. J. 289, 425. DOI. ADS.

Fisher, G.H., Canfield, R.C., McClymont, A.N.: 1985c, Flare loop radiative hydrodynamics. V - Response to thick-target heating. VI - Chromospheric evaporation due to heating by nonthermal electrons. VII - Dynamics of the thick-target heated chromosphere. Astrophys. J. 289, 414. DOI. ADS.

Fisher, G.H., Canfield, R.C., McClymont, A.N.: 1985d, Flare loop radiative hydrodynamics. V - Response to thick-target heating. VI - Chromospheric evaporation due to heating by nonthermal electrons. VII - Dynamics of the thick-target heated chromosphere. Astrophys. J. 289, 414. DOI. ADS.

Fisher, G.H., Bercik, D.J., Welsch, B.T., Hudson, H.S.: 2012, Global Forces in Eruptive Solar Flares: The Lorentz Force Acting on the Solar Atmosphere and the Solar Interior. Solar Phys. 277, 59. DOI. ADS.

Gordovskyy, M., Zharkova, V.V., Voitenko, Y.M., Goossens, M.: 2005, Proton versus electron heating in solar flares. Adv. in Space Res. 35, 1743. DOI. ADS.

Holman, G.D., Sui, L., Schwartz, R.A., Emslie, A.G.: 2003, Electron Bremsstrahlung Hard X-Ray Spectra, Electron Distributions, and Energetics in the 2002 July 23 Solar Flare. Astrophys. J. Lett. 595, L97. DOI. ADS.

Hudson, H.S.: 1972, Thick-Target Processes and White-Light Flares. Solar Phys. 24, 414. DOI. ADS.

Hudson, H.S., Fisher, G.H., Welsch, B.T.: 2008, Flare Energy and Magnetic Field Variations. In: Howe, R., Komm, R.W., Balasubramaniam, K.S., Petrie, G.J.D. (eds.) Subsurface and Atmospheric Influences on Solar Activity, Astron. Soc. Pacific C.S. 383, 221. ADS.

Kitiashvili, I.N., Kosovichev, A.G., Mansour, N.N., Wray, A.A.: 2011, Excitation of Acoustic Waves by Vortices in the Quiet Sun. Astrophys. J. Lett. 727, L50. DOI. ADS.

Kobylinskii, V.A., Zharkova, V.V.: 1996, Hydrogen H-alpha line emissions and radiative losses in the impulsive solar events. Adv. Space Res. 17. DOI. ADS.

Kosovichev, A.G.: 2006, Properties of Flares-Generated Seismic Waves on the Sun. Solar Phys. 238, 1. DOI. ADS.

Kosovichev, A.G.: 2007, The Cause of Photospheric and Helioseismic Responses to Solar Flares: High-Energy Electrons or Protons? Astrophys. J. Lett. 670, L65. DOI. ADS. 
Kosovichev, A.G., Zharkova, V.V.: 1995, Seismic Response to Solar Flares: Theoretical Predictions. In: Helioseismology, ESA S.P. 376, 341. ADS.

Kosovichev, A.G., Zharkova, V.V.: 1998, X-ray flare sparks quake inside Sun. Nature 393, 317. DOI. ADS.

Kosovichev, A.G., Zharkova, V.V.: 1999, Variations of Photospheric Magnetic Field Associated with Flares and CMEs. Solar Phys. 190, 459. DOI. ADS.

Kosovichev, A.G., Zharkova, V.V.: 2001, Magnetic Energy Release and Transients in the Solar Flare of 2000 July 14. Astrophys. J. Lett. 550, L105. DOI. ADS.

Lindsey, C., Braun, D.C.: 1997, Helioseismic Holography. Astrophys. J. 485, 895. ADS.

Lindsey, C., Braun, D.C.: 2000, Basic Principles of Solar Acoustic Holography - (Invited Review). Solar Phys. 192, 261. ADS.

Lindsey, C., Donea, A.: 2008, Mechanics of Seismic Emission from Solar Flares. Solar Phys. 251, 627. DOI. ADS.

Litvinenko, Y.E.: 1996, Particle Acceleration in Reconnecting Current Sheets with a Nonzero Magnetic Field. Astrophys. J. 462, 997. DOI. ADS.

Machado, M.E., Emslie, A.G., Avrett, E.H.: 1989, Radiative backwarming in white-light flares. Solar Phys. 124, 303. DOI. ADS.

Martínez-Oliveros, J.C., Donea, A.-C.: 2009, Magnetic field variations and seismicity of solar active regions. Mon. Not. Roy. Astron. Soc. 395, L39. DOI. ADS.

Martínez-Oliveros, J.C., Moradi, H., Donea, A.: 2008, Seismic Emissions from a Highly Impulsive M6.7 Solar Flare. Solar Phys. 251, 613. DOI. ADS.

Martínez Oliveros, J.-C., Hudson, H.S., Hurford, G.J., Krucker, S., Lin, R.P., Lindsey, C., Couvidat, S., Schou, J., Thompson, W.T.: 2012, The Height of a White-light Flare and Its Hard X-Ray Sources. Astrophys. J. Lett. 753, L26. DOI. ADS.

Matthews, S.A., Zharkov, S., Zharkova, V.V.: 2011, Anatomy of a Solar Flare: Measurements of the 2006 December 14 X-class Flare with GONG, Hinode, and RHESSI. Astrophys. J. 739, 71. DOI. ADS.

Mauas, P.J.D., Gómez, D.O.: 1997, Fokker-Planck Description of Electron Beams in the Solar Chromosphere. Astrophys. J. 483, 496. ADS.

Metcalf, T.R., Alexander, D., Hudson, H.S., Longcope, D.W.: 2003, TRACE and Yohkoh Observations of a White-Light Flare. Astrophys. J. 595, 483. DOI. ADS.

Moll, R., Cameron, R.H., Schüssler, M.: 2011, Vortices in simulations of solar surface convection. Astron. Astrophys. 533, A126. DOI. ADS.

Nagai, F., Emslie, A.G.: 1984, Gas dynamics in the impulsive phase of solar flares. I Thicktarget heating by nonthermal electrons. Astrophys. J. 279, 896. DOI. ADS.

Norton, A.A., Graham, J.P., Ulrich, R.K., Schou, J., Tomczyk, S., Liu, Y., Lites, B.W., López Ariste, A., Bush, R.I., Socas-Navarro, H., Scherrer, P.H.: 2006, Spectral Line Selection for HMI: A Comparison of Fe I $6173 \AA$ and Ni I $6768 \AA$. Solar Phys. 239, 69. DOI. ADS.

Petrie, G.J.D.: 2012, The Abrupt Changes in the Photospheric Magnetic and Lorentz Force Vectors during Six Major Neutral-line Flares. Astrophys. J. 759, 50. DOI. ADS.

Petrie, G.J.D.: 2013, A Spatio-temporal Description of the Abrupt Changes in the Photospheric Magnetic and Lorentz-Force Vectors During the 15 February 2011 X2.2 Flare. Solar Phys. 287, 415. DOI. ADS.

Petrie, G.J.D., Sudol, J.J.: 2010, Abrupt Longitudinal Magnetic Field Changes in Flaring Active Regions. Astrophys. J. 724, 1218. DOI. ADS.

Priest, E.R., Forbes, T.G.: 2002, The magnetic nature of solar flares. Astron. Astrophys. Rev. 10, 313. DOI. ADS.

Qiu, J., Gary, D.E.: 2003, Flare-related Magnetic Anomaly with a Sign Reversal. Astrophys. J. 599, 615. DOI. ADS.

Qiu, J., Lee, J., Gary, D.E., Wang, H.: 2002, Motion of Flare Footpoint Emission and Inferred Electric Field in Reconnecting Current Sheets. Astrophys. J. 565, 1335. DOI. ADS.

Siversky, T.V., Zharkova, V.V.: 2009a, Particle acceleration in a reconnecting current sheet: PIC simulation. J. Plasma Phys. 75, 619. DOI. ADS.

Siversky, T.V., Zharkova, V.V.: 2009b, Stationary and impulsive injection of electron beams in converging magnetic field. Astron. Astrophys. 504, 1057. DOI. ADS.

Somov, B.V. (ed.): 2000, Cosmic Plasma Physics, Astrophys. Space Scien. Lib. 251. ADS.

Somov, B.V., Spektor, A.R., Syrovatskii, S.I.: 1981, Hydrodynamic response of the solar chromosphere to an elementary flare burst. I - Heating by accelerated electrons. Solar Phys. 73, 145. DOI. ADS

Sudol, J.J., Harvey, J.W.: 2005, Longitudinal Magnetic Field Changes Accompanying Solar Flares. Astrophys. J. 635, 647. DOI. ADS. 
Syrovatskii, S.I., Shmeleva, O.P.: 1972, Heating of Plasma by High-Energy Electrons, and Nonthermal X-Ray Emission in Solar Flares. Soviet Astron. 16, 273. ADS.

van den Oord, G.H.J.: 1990, The electrodynamics of beam/return current systems in the solar corona. Astron. Astrophys. 234, 496. ADS.

Vernazza, J.E., Avrett, E.H., Loeser, R.: 1981, Structure of the solar chromosphere. III - Models of the EUV brightness components of the quiet-sun. Astrophys. J. Suppl. Series 45, 635. DOI. ADS.

Wang, S., Liu, C., Liu, R., Deng, N., Liu, Y., Wang, H.: 2012, Response of the Photospheric Magnetic Field to the X2.2 Flare on 2011 February 15. Astrophys. J. Lett. 745, L17. DOI. ADS.

Warmuth, A., Mann, G.: 2011, Kinematical evidence for physically different classes of largescale coronal EUV waves. Astron. Astrophys. 532, A151. DOI. ADS.

Wolff, C.L.: 1972, Free Oscillations of the Sun and Their Possible Stimulation by Solar Flares. Astrophys. J. 176, 833. DOI. ADS.

Zarro, D.M., Slater, G.L., Freeland, S.L.: 1988, Impulsive phase soft X-ray blueshifts at a loop footpoint. Astrophys. J. Lett. 333, L99. DOI. ADS.

Zarro, D.M., Canfield, R.C., Metcalf, T.R., Strong, K.T.: 1988, Explosive plasma flows in a solar flare. Astrophys. J. 324, 582. DOI. ADS.

Zharkov, S.: 2013, Geometric properties of acoustic waves generated by a point source in the solar-like interior: effects of acoustic cutoff frequency. Mon. Not. Roy. Astron. Soc. 431, 3414. DOI. ADS.

Zharkov, S., Zharkova, V.V., Matthews, S.A.: 2011, Comparison of Seismic Signatures of Flares Obtained by SOHO/Michelson Doppler Imager and GONG Instruments. Astrophys. J. 739, 70. DOI. ADS.

Zharkov, S., Green, L.M., Matthews, S.A., Zharkova, V.V.: 2011, 2011 February 15: Sunquakes Produced by Flux Rope Eruption. Astrophys. J. Lett. 741, L35. DOI. ADS.

Zharkov, S., Green, L.M., Matthews, S.A., Zharkova, V.V.: 2013, Properties of the 15 February 2011 Flare Seismic Sources. Solar Phys. 284, 315. DOI. ADS.

Zharkova, V.V.: 2008, The Mechanisms of Particle Kinetics and Dynamics Leading to Seismic Emission and Sunquakes. Solar Phys. 251, 641. DOI. ADS.

Zharkova, V.V., Agapitov, O.V.: 2009, The effect of magnetic topology on particle acceleration in a three-dimensional reconnecting current sheet: a test-particle approach. J. Plasma Phys. 75, 159. DOI. ADS.

Zharkova, V.V., Gordovskyy, M.: 2004, Particle Acceleration Asymmetry in a Reconnecting Nonneutral Current Sheet. Astrophys. J. 604, 884. DOI. ADS.

Zharkova, V.V., Gordovskyy, M.: 2005, Energy spectra of particles accelerated in a reconnecting current sheet with the guiding magnetic field. Mon. Not. Roy. Astron. Soc. 356, 1107. DOI. ADS.

Zharkova, V.V., Gordovskyy, M.: 2006, The Effect of the Electric Field Induced by Precipitating Electron Beams on Hard X-Ray Photon and Mean Electron Spectra. Astrophys. J. 651, 553. DOI. ADS.

Zharkova, V.V., Khabarova, O.V.: 2012, Particle Dynamics in the Reconnecting Heliospheric Current Sheet: Solar Wind Data versus Three-dimensional Particle-in-cell Simulations. Astrophys. J. 752, 35. DOI. ADS.

Zharkova, V.V., Kobylinskii, V.A.: 1993, The effect of non-thermal excitation and ionization on the hydrogen emission in impulsive solar flares. Solar Phys. 143, 259. DOI. ADS.

Zharkova, V.V., Kosovichev, A.G.: 2000, Helioseismic Waves and Magnetic Field Variations Induced by Solar Flares as Probes of Energy Transport Mechanisms. In: Ramaty R.\& Mandzhavidze N. (eds.) High Energy Solar Physics Workshop - Anticipating Hess!, Astron. Soc. Pacific C.S. 206, 77. ADS.

Zharkova, V.V., Kosovichev, A.G.: 2002, A new insight into the energy release and transport in solar flares. In: H. Sawaya-Lacoste (ed.) Solspa 2001, Proceedings of the Second Solar Cycle and Space Weather Euroconference, ESA Special Publication 477, 35. ADS.

Zharkova, V.V., Siversky, T.: 2011, Formation of electron clouds during particle acceleration in a 3D current sheet. In: Bonanno, A., de Gouveia Dal Pino, E., Kosovichev, A.G. (eds.) IAU Symposium, IAU Symposium 274, 453. DOI. ADS.

Zharkova, V.V., Zharkov, S.I.: 2007, On the Origin of Three Seismic Sources in the Proton-rich Flare of 2003 October 28. Astrophys. J. 664, 573. DOI. ADS.

Zharkova, V.V., Zharkov, S.I., Ipson, S.S., Benkhalil, A.K.: 2005, Toward magnetic field dissipation during the 23 July 2002 solar flare measured with Solar and Heliospheric Obser- 
vatory/Michelson Doppler Imager (SOHO/MDI) and Reuven Ramaty High Energy Solar Spectroscopic Imager (RHESSI). J. Geophys. Res. 110, 8104. DOI. ADS.

Zharkova, V.V., Arzner, K., Benz, A.O., Browning, P., Dauphin, C., Emslie, A.G., Fletcher, L., Kontar, E.P., Mann, G., Onofri, M., Petrosian, V., Turkmani, R., Vilmer, N., Vlahos, L.: 2011a, Recent Advances in Understanding Particle Acceleration Processes in Solar Flares. Space Sci. Rev. 159, 357. DOI. ADS.

Zharkova, V.V., Kashapova, L.K., Chornogor, S.N., Andrienko, O.V.: 2011b, The effect of energetic particle beams on the chromospheric emission of the 2004 July 25 flare. Mon. Not. Roy. Astron. Soc. 411, 1562. DOI. ADS. 